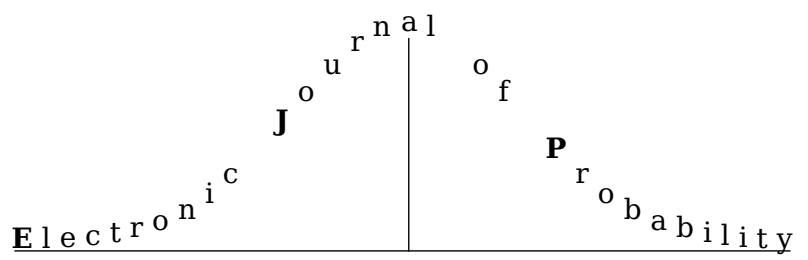

Electron. J. Probab. 25 (2020), no. 50, 1-22.

ISSN: 1083-6489 https://doi.org/10.1214/20-EJP448

\title{
Stochastic partial integral-differential equations with divergence terms*
}

\author{
Chi Hong Wong ${ }^{\dagger} \quad$ Xue Yang $^{\ddagger} \quad$ Jing Zhang ${ }^{\S}$
}

\begin{abstract}
We study a class of stochastic partial integral-differential equations with an asymmetrical non-local operator $\frac{1}{2} \Delta+a^{\alpha} \Delta^{\frac{\alpha}{2}}+b \cdot \nabla$ and a distribution expressed as divergence of a measurable field. For $0<\alpha<2$, the existence and uniqueness of solution is proved by analytical method, and a probabilistic interpretation, similar to the Feynman-Kac formula, is presented for $0<\alpha<1$. The method of backward doubly stochastic differential equations is also extended in this work.
\end{abstract}

Keywords: stochastic partial integral-differential equations; non-local operator; fractional Laplacian; forward-backward martingale decomposition; backward doubly stochastic differential equations.

AMS MSC 2010: 60H15; 60G46; 35R60.

Submitted to EJP on October 4, 2018, final version accepted on April 6, 2020.

\section{Introduction}

We consider the following stochastic partial integral-differential equation in this article

$$
\left\{\begin{array}{c}
d u_{t}(x)+\left[\mathcal{A} u_{t}(x)+f_{t}\left(x, u_{t}(x), \nabla u_{t}(x)\right)\right] d t+h_{t}\left(x, u_{t}(x), \nabla u_{t}(x)\right) \overleftarrow{d B_{t}} \\
\quad+\operatorname{div} g_{t}\left(x, u_{t}(x), \nabla u_{t}(x)\right) d t=0, \quad(t, x) \in[0, T] \times \mathbb{R}^{d} \\
u_{T}(x)=\Phi(x), \quad x \in \mathbb{R}^{d} .
\end{array}\right.
$$

For constants $a>0$ and $0<\alpha<2$, the non-local operator $\mathcal{A}=\frac{1}{2} \Delta+a^{\alpha} \Delta^{\frac{\alpha}{2}}+b \cdot \nabla$ is a non-symmetric infinitesimal generator of a Markov process with jumps. Coefficients $f$, $g=\left(g_{1}, \cdots, g_{d}\right)$ and $h=\left(h_{1}, \cdots, h_{d^{1}}\right)$ are non-linear random functions. The differential term with $\overleftarrow{d B}_{t}$ refers to a backward stochastic integral with respect to a $d^{1}$-dimensional

\footnotetext{
*Supported by National Natural Science Foundation of China $(11401427,11401108,11771329,11871052$, 11861029) and Shanghai Science and Technology Commission Grant (14PJ1401500).

${ }^{\dagger}$ Tianjin University, China. E-mail: chiwong@tju.edu.cn

${ }^{\ddagger}$ Tianjin University, China. E-mail: xyang2013@tju.edu.cn

${ }^{\S}$ Fudan University, China. E-mail: zhang_jing@fudan.edu.cn
} 
Brownian motion on probability space $\left(\Omega^{\prime}, \mathcal{F}^{B}, \mathbb{P}^{\prime},\left(B_{t}\right)_{t \geq 0}\right)$, so that the doubly stochastic framework introduced by Pardoux and Peng [15] could be applied.

This article is devoted to extending the current methods of stochastic partial integraldifferential equations (PIDEs for short) and backward doubly stochastic differential equations (BDSDEs for short) driven by some Lévy processes, while a singular term " $\operatorname{div} g$ " is involved in the equation which is understood in distributional sense. Throughout this paper a classic Sobolev weak solution is considered. The contribution of this study is threefold: types of forward-backward martingale decomposition and Fukushima decomposition, which correspond to an asymmetric Markov process with jumps, are presented respectively; a stochastic representation, similar to Feymann-Kac formula, for the solution of stochastic PIDE (1.1) is obtained; a connection between stochastic PIDEs with singular terms and a class of BDSDEs is built. In a special case that $h \equiv 0$, equation (1.1) turns out to be a parabolic partial differential equation (PDE for short). Then actually, the connection between this PDE and some backward stochastic differential equation (BSDE for short) is also obtained, which generalizes the classic connection between PDEs and BSDEs (see $[13,14,16]$ ). In particular, we prove the existence and uniqueness of solution to the stochastic PIDEs by analytical approach in Section 4.1. This is a generalization of the result in [5], where only symmetric operator was considered. From this point of view, Section 4.1 has its own independent interest.

Inspired by a serial of works (see, for example, $[5,8,10,18]$ ) on dealing with the singular term $\operatorname{div} g$, we consider a stochastic PIDE with non-local operator that is associated with a perturbed Lévy process $\left(X_{t}\right)_{t \geq 0}$. As a generalization of the results in $[8,18]$, we consider the non-local operator and define a stochastic *-integral for $u$, when $u$ is in the domain of the bilinear form $\mathcal{E}$ associated with $\mathcal{A}$. Precisely, for $u \in \mathcal{D}(\mathcal{A})$,

$$
\int_{s}^{t} \nabla u * d X:=\left.M u\right|_{s} ^{t}+\left.\bar{M} u\right|_{s} ^{t}+\int_{s}^{t} 2 a^{\alpha} \Delta^{\frac{\alpha}{2}} u\left(X_{r}\right)+\frac{\mathcal{G}\left(u, p_{r}^{\mu}\right)}{p_{r}^{\mu}}\left(X_{r}\right) d r,
$$

where the forward and backward martingales are included. The last term on the right hand side comes from the asymmetry of $\mathcal{A}$, and it may vanish when a symmetric operator is considered, since $p^{m} \equiv 1$ if the perturbation $b=0$ (see Section 3.1).

Moreover, a further decomposition of the zero-energy part of Fukushima decomposition of $u\left(X_{t}\right)$ is given in Proposition 3.8. It holds that

$$
u\left(X_{t}\right)-u\left(X_{s}\right)=\left.M u\right|_{s} ^{t}+\int_{s}^{t} a^{\alpha} \Delta^{\frac{\alpha}{2}} u\left(X_{r}\right)+\langle b, \nabla u\rangle\left(X_{r}\right) d r-\frac{1}{2} \int_{s}^{t} \nabla u * d X, \quad \text { for } s<t .
$$

Due to this decomposition, we consider the solution $u$ to stochastic PIDE (1.1), of which the existence and uniqueness is proved in Theorem 4.2, and find that the process $u_{t}\left(X_{t}\right)$ gives a solution to a class of BDSDEs,

$$
\begin{aligned}
Y_{t}=\Phi\left(X_{T}\right) & -\int_{t}^{T} Z_{r} d W_{r}-\int_{t}^{T} \int_{\mathbb{R}^{d}} U(r, z) \tilde{N}(d z, d r)+\int_{t}^{T} h\left(r, X_{r}, Y_{r}, Z_{r}\right) \overleftarrow{d B}_{r} \\
& +\int_{t}^{T} f\left(r, X_{r}, Y_{r}, Z_{r}\right) d r-\int_{t}^{T} g\left(r, X_{r}, Y_{r}, Z_{r}\right) * d X
\end{aligned}
$$

which involves a stochastic *-integral, driven by a Brownian motion and a compensated Poisson process. Conversely, we prove that this BDSDE can also provide a candidate solution for stochastic PIDE (1.1), which can be seen as a well-known application of BDSDEs (see Theorem 5.1).

By the expression of stochastic *-integral, it can be seen that $u$ needs to be in the domain of fractional Laplacian operator, which is known as fractional Sobolev space $H^{\alpha}\left(\mathbb{R}^{d}\right)$. That is the reason why we ask $\alpha$ to take values between 0 and 1 . We will 
leave the problem when $\alpha \in(1,2)$ to our future work. We also want to mention that the existence and uniqueness of solution to stochastic PIDE hold for $0<\alpha<2$, since analytical methods are applied there.

The paper is organized as follows. In Section 2, we introduce the basic function spaces, Dirichlet forms and stochastic processes. Section 3 is the main part of the paper in which we define the stochastic *-integral and prove the forward-backward martingale decomposition corresponding to the non-symmetric non-local generator. Then, in Section 4 , we prove the existence and uniqueness of solution to the stochastic PIDEs and give a probabilistic interpretation for this solution. The last section is devoted to building the connection between stochastic PIDEs and BDSDEs.

\section{Preliminaries}

Throughout this paper, we assume $d \geq 1$ as an integer. Let $L^{2}\left(\mathbb{R}^{d}\right)$ be the space of square integrable functions with respect to Lebesgue measure on $\mathbb{R}^{d}$, which is a Hilbert space equipped with the inner product and norm

$$
(u, v):=\int_{\mathbb{R}^{d}} u(x) v(x) d x, \quad\|u\|:=\left(\int_{\mathbb{R}^{d}} u^{2}(x) d x\right)^{\frac{1}{2}}, u, v \in L^{2}\left(\mathbb{R}^{d}\right) .
$$

Since an evolution problem over a fixed time interval $[0, T]$ will be considered, we define the norm for a function in Hilbert space $L^{2}\left([0, T] \times \mathbb{R}^{d}\right)$ as

$$
\|u\|_{2,2}:=\left(\int_{0}^{T} \int_{\mathbb{R}^{d}}|u(t, x)|^{2} d x d t\right)^{\frac{1}{2}}
$$

Let $H^{1}\left(\mathbb{R}^{d}\right):=\left\{u: u \in L^{2}\left(\mathbb{R}^{d}\right),|\nabla u| \in L^{2}\left(\mathbb{R}^{d}\right)\right\}$ be the first order Sobolev space equipped with inner product and norm

$$
(u, v)_{H^{1}\left(\mathbb{R}^{d}\right)}:=(u, v)+(\nabla u, \nabla v), \quad\|u\|_{H^{1}\left(\mathbb{R}^{d}\right)}:=\left(\|u\|^{2}+\|\nabla u\|^{2}\right)^{\frac{1}{2}},
$$

where $\nabla u(x):=\left(\partial_{1} u(x), \cdots, \partial_{d} u(x)\right)$ is gradient of function $u$.

Let $\widetilde{F}$ be the space of all functions $u \in L^{2}\left([0, T] ; H^{1}\left(\mathbb{R}^{d}\right)\right)$ such that $t \mapsto u_{t}=u(t, \cdot)$ is continuous in $L^{2}\left(\mathbb{R}^{d}\right)$, which is a Banach space equipped with the norm as follows,

$$
\|u\|_{T}^{2}:=\sup _{0 \leq t \leq T}\left\|u_{t}\right\|^{2}+\int_{0}^{T}\left\|\nabla u_{t}\right\|^{2} d t
$$

The space of test functions is denoted by $\mathcal{D}_{T}=\mathcal{C}^{\infty}([0, T]) \otimes \mathcal{C}_{c}^{\infty}\left(\mathbb{R}^{d}\right)$, where $\mathcal{C}^{\infty}([0, T])$ is the space of real functions which can be extended as infinite differential functions in the neighborhood of $[0, T]$ and $\mathcal{C}_{c}^{\infty}\left(\mathbb{R}^{d}\right)$ is the space of infinite differentiable functions with compact support in $\mathbb{R}^{d}$.

For $\alpha \in(0,2)$, the fractional Laplacian $\Delta^{\frac{\alpha}{2}}$, which can be seen as the most basic integral-differential operator, can be defined in several equivalent ways (see [7]). For any $f \in C_{c}^{\infty}\left(\mathbb{R}^{d}\right)$, it is defined as follows

$$
\Delta^{\frac{\alpha}{2}} f(x):=\lim _{\epsilon \rightarrow 0} \int_{|y| \geq \epsilon} C_{d, \alpha}(f(y)-f(x)) \frac{d y}{|y-x|^{d+\alpha}},
$$

where the normalizing constant is $C_{d, \alpha}:=\frac{\alpha 2^{\alpha-1} \Gamma\left(\frac{d+\alpha}{2}\right)}{\pi^{\frac{d}{2}} \Gamma\left(1-\frac{\alpha}{2}\right)}$. Let $\mathbf{F}$ and $\mathbf{F}^{-1}$ denote Fourier and inverse Fourier transform on $L^{2}\left(\mathbb{R}^{d}\right)$ respectively. It is known that Fourier transformation of $\Delta^{\frac{\alpha}{2}}$ satisfies

$$
\mathbf{F}\left(\Delta^{\frac{\alpha}{2}} f\right)(\xi)=|\xi|^{\alpha} \mathbf{F}(f)(\xi), \quad \xi \in \mathbb{R}^{d} .
$$


For $s>0$, let

$$
H^{s}\left(\mathbb{R}^{d}\right):=\left\{f \in L^{2}\left(\mathbb{R}^{d}\right): \mathbf{F}^{-1}\left[\left(1+|\xi|^{2}\right)^{\frac{s}{2}} \mathbf{F} f\right] \in L^{2}\left(\mathbb{R}^{d}\right)\right\}
$$

be the Sobolev space of order $s$, which is equipped with the norm

$$
\|f\|_{H^{s}}:=\left\|\mathbf{F}^{-1}\left(1+|\xi|^{2}\right)^{\frac{s}{2}} \mathbf{F} f\right\| \text {. }
$$

By interpolation and Sobolev embedding theorem (see [20]), for $0<s<s^{\prime}$, it holds that

$$
H^{s^{\prime}}\left(\mathbb{R}^{d}\right) \subset H^{s}\left(\mathbb{R}^{d}\right) \subset L^{2}\left(\mathbb{R}^{d}\right) .
$$

It is known that $\Delta^{\frac{\alpha}{2}}$ maps $H^{\alpha}\left(\mathbb{R}^{d}\right)$ into $L^{2}\left(\mathbb{R}^{d}\right)$. In other words, the domain of $\Delta^{\frac{\alpha}{2}}$ satisfies that $\mathcal{D}\left(\Delta^{\frac{\alpha}{2}}\right)=H^{\alpha}\left(\mathbb{R}^{d}\right)$.

For any $\gamma>0$, we define the Kato class

$$
\mathcal{K}_{\gamma}:=\left\{\mu(d x): \lim _{\epsilon \rightarrow 0} \sup _{x \in \mathbb{R}^{d}} \int_{B(x, \epsilon)} \frac{1}{|x-y|^{\gamma}}|\mu|(d y)=0\right\},
$$

where $|\mu(d x)|$ is the total variation of the signed measure $\mu(d x)$ and $B(x, \epsilon)$ is the ball in $\mathbb{R}^{d}$ centred at $x$ with radius $\epsilon$. We say a function $f \in \mathcal{K}_{\gamma}$ if the signed measure $\mu(d x)=f(x) d x \in \mathcal{K}_{\gamma}$. It is easy to check that, if $d>2$, for any function $|f| \in L^{p}\left(\mathbb{R}^{d}\right)$, $p>d$, then $|f| \in \mathcal{K}_{d-1}$ and $|f|^{2} \in \mathcal{K}_{d-2}$.

We assume that $b:=\left(b_{1}, \ldots, b_{d}\right): \mathbb{R}^{d} \rightarrow \mathbb{R}^{d}$ satisfies $|b|^{2} \in \mathcal{K}_{d-2}$ for $d>2$ and $|b| \in L^{\infty}\left(\mathbb{R}^{d}\right)$ otherwise. Consider a non-symmetric bilinear form $\left(\mathcal{E}, H^{1}\left(\mathbb{R}^{d}\right)\right)$ on $L^{2}\left(\mathbb{R}^{d}\right)$ defined as follows,

$$
\mathcal{E}(u, v):=\mathcal{E}^{1}(u, v)+\mathcal{E}^{2}(u, v)-\int_{\mathbb{R}^{d}}\langle b(x), \nabla u(x)\rangle v(x) d x
$$

with

$$
\mathcal{E}^{1}(u, v):=\frac{1}{2}(\nabla u, \nabla v), \quad \mathcal{E}^{2}(u, v):=\frac{a^{\alpha} C_{d, \alpha}}{2} \int_{\mathbb{R}^{d} \times \mathbb{R}^{d} \backslash \Gamma} \frac{(u(x)-u(y))(v(x)-v(y))}{|x-y|^{d+\alpha}} d x d y,
$$

where $\Gamma$ is the diagonal $\Gamma:=\left\{(x, x) \mid x \in \mathbb{R}^{d}\right\}$ and $\alpha \in(0,2)$. For convenience, we use the notation $\mathcal{E}(u):=\mathcal{E}(u, u)$, for $u \in H^{1}\left(\mathbb{R}^{d}\right)$.

By [3] and [19], we know that the bilinear form $\left(\mathcal{E}, H^{1}\left(\mathbb{R}^{d}\right)\right)$ is a lower-bounded regular Dirichlet form and is related to a Hunt process $\left(X_{t}\right)_{t \geq 0}$ whose infinitesimal generator $\mathcal{A}$ is of the following form

$$
\mathcal{A} u:=L u+K u+b \cdot \nabla u,
$$

with

$$
L u:=\frac{1}{2} \Delta u \quad \text { and } \quad K u:=a^{\alpha} \Delta^{\frac{\alpha}{2}} u .
$$

In fact, by Theorem 3.25 in [3], for any $v \in H^{1}\left(\mathbb{R}^{d}\right)$ and $\epsilon \in(0,1)$, there exists $C(\epsilon)>0$ such that

$$
\int_{\mathbb{R}^{d}}|b(x)|^{2}|v(x)|^{2} d x \leq \epsilon \int_{\mathbb{R}^{d}}|\nabla v(x)|^{2} d x+C(\epsilon) \int_{\mathbb{R}^{d}}|v(x)|^{2} d x .
$$

Then, for any $u, v \in H^{1}\left(\mathbb{R}^{d}\right)$, by Hölder's inequality and Kato-type inequality (2.1), one has

$$
\begin{aligned}
\left|\int_{\mathbb{R}^{d}}\langle b(x), \nabla u(x)\rangle v(x) d x\right| & \leq\left(\int_{\mathbb{R}^{d}}|\nabla u(x)|^{2} d x\right)^{\frac{1}{2}}\left(\epsilon \int_{\mathbb{R}^{d}}|\nabla v(x)|^{2} d x+C(\epsilon) \int_{\mathbb{R}^{d}}|v(x)|^{2} d x\right)^{\frac{1}{2}} \\
& \leq \sqrt{\epsilon \vee C(\epsilon)}\|u\|_{H^{1}\left(\mathbb{R}^{d}\right)} \cdot\|v\|_{H^{1}\left(\mathbb{R}^{d}\right)},
\end{aligned}
$$


where $\epsilon \in(0,1)$ and $C(\epsilon)>0$. By the first inequality in (2.2) and Sobolev embedding theorem, there exists a constant $\kappa_{0}>0$ and $\delta>0$ such that for any $\kappa>\kappa_{0}$,

$$
\mathcal{E}(u, u)+\kappa(u, u) \geq \delta\|u\|_{H^{1}\left(\mathbb{R}^{d}\right)}^{2}
$$

Furthermore, by Sovolev embedding theorem and the second inequality in (2.2), there exists a constant $M>0$ such that

$$
|\mathcal{E}(u, v)| \leq M\|u\|_{H^{1}\left(\mathbb{R}^{d}\right)} \cdot\|v\|_{H^{1}\left(\mathbb{R}^{d}\right)} .
$$

Therefore, $\left(\mathcal{E}, H^{1}\left(\mathbb{R}^{d}\right)\right)$ is a well-defined closed form on $L^{2}\left(\mathbb{R}^{d}\right)$, and by [11], there are unique strong continuous semigroups $\left(P_{t}\right)_{t \geq 0}$ and $\left(P_{t}^{*}\right)_{t \geq 0}$ with

$$
\left(P_{t} f, g\right)=\left(f, P_{t}^{*} g\right), \quad \forall f, g \in L^{2}\left(\mathbb{R}^{d}\right)
$$

of which the corresponding generators $\mathcal{A}$ and $\mathcal{A}^{*}$ satisfying

$$
\mathcal{E}(u, v)=(-\mathcal{A} u, v)=\left(u,-\mathcal{A}^{*} v\right), \quad \text { for } u \in \mathcal{D}(\mathcal{A}) \text { and } v \in \mathcal{D}\left(\mathcal{A}^{*}\right)
$$

It is known that the transition function is absolutely continuous with respect to the Lebesgue measure, i.e.

$$
P_{t}(x, d y)=p(t, x, y) d y,
$$

where $p(t, x, y)$ is the density. By [2], when $|b| \in \mathcal{K}_{d-1}$, the heat kernel $p(t, x, y)$ is continuous on $\mathbb{R}^{+} \times \mathbb{R}^{d} \times \mathbb{R}^{d}$ and for any $t>0$ and $x \in \mathbb{R}^{d}, \int_{\mathbb{R}^{d}} p(t, x, y) d y=1$. Moreover, the following two-sided estimate holds: there exist constants $C_{i}, i=1,2,3,4$ such that

$$
C_{3}\left[t^{-\frac{d}{2}} e^{-\frac{C_{4}|x-y|^{2}}{t}}+t^{\frac{d}{2}} \wedge \frac{t}{|x-y|^{d+\alpha}}\right] \leq p(t, x, y) \leq C_{1}\left[t^{-\frac{d}{2}} e^{-\frac{C_{2}|x-y|^{2}}{t}}+t^{\frac{d}{2}} \wedge \frac{t}{|x-y|^{d+\alpha}}\right] .
$$

Let $D\left(\mathbb{R}^{+} ; \mathbb{R}^{d}\right)$ be the space of right continuous $\mathbb{R}^{d}$-valued functions on $\mathbb{R}^{+}$having left limits equipped with Skorokhod topology. There is a Lévy process $\left(\Omega, X_{t}, \theta_{t}, \mathcal{F}, \mathcal{F}_{t}, \mathbb{P}^{x}, x \in\right.$ $\left.\mathbb{R}^{d}\right)$ associated to the Dirichlet form $\left(\mathcal{E}, H^{1}\left(\mathbb{R}^{d}\right)\right)$ on canonical paths space $\Omega:=D\left(\mathbb{R}^{+} ; \mathbb{R}^{d}\right)$. For the process $X$, we have decomposition

$$
X_{t}=X_{0}+W_{t}+\int_{0}^{t} b\left(X_{s}\right) d s+\int_{0}^{t} \int_{|z| \geq 1} z N(d z, d s)+\int_{0}^{t} \int_{|z|<1} z \tilde{N}(d z, d s)
$$

where $\left(W_{t}\right)_{t>0}$ is a $d$-dimensional standard Brownian motion, $N(d z, d t)$ is the jumping measure of $X$ with $\nu(d z) d t:=\frac{a^{\alpha} C_{d, \alpha}}{|z|^{\mid+\alpha}} d z d t$ as its predictable compensator and $\tilde{N}(d z, d t):=$ $N(d z, d t)-\nu(d z) d t$ is the associated compensated random jump measure.

For any $u \in H^{1}\left(\mathbb{R}^{d}\right)$, we have Fukushima decomposition (see Theorem 5.1.5, [12]),

$$
u\left(X_{t}\right)-u\left(X_{s}\right)=\left.M u\right|_{s} ^{t}+\left.N u\right|_{s} ^{t}
$$

where $\left.M u\right|_{s} ^{t}:=\int_{s}^{t}\left\langle\nabla u\left(X_{r}\right), d W_{r}\right\rangle+\int_{s}^{t} \int_{\mathbb{R}^{d}}\left[u\left(X_{r-}+z\right)-u\left(X_{r-}\right)\right] \tilde{N}(d z, d r)$ is the martingale additive functional and $\left.N u\right|_{s} ^{t}$ is the zero-energy additive functional. For any function $u$ in the domain of generator $\mathcal{A}$, i.e., $u \in \mathcal{D}(\mathcal{A})$, it is known that $\left.N u\right|_{s} ^{t}=\int_{s}^{t} \mathcal{A} u\left(X_{r}\right) d r$.

\section{Decomposition with forward and backward martingales}

In this section, we give a forward-backward martingale decomposition corresponding to the asymmetric non-local operator $\mathcal{A}$, for which a stochastic *-integral is defined. 
Consider the reverse process $\left(X_{T-t}\right)_{t \in[0, T]}$ under the probability $\mathbb{P}^{o}$, for fixed $o \in \mathbb{R}^{d}$, with the non-homogenous transition function

$$
Q_{0, t} u(x):=\frac{\int_{\mathbb{R}^{d}} p(T-t, o, y) u(y) p(t, y, x) d y}{p(T, o, x)} .
$$

We denote the density of $Q_{0, t}$ by $p_{Q}(t, x, y):=\frac{p(T-t, o, y) p(t, y, x)}{p(T, o, x)}$.

Set

$$
\begin{aligned}
J(u, v)(x): & =\int_{\mathbb{R}^{d}-\{0\}} a^{\alpha} C_{d, \alpha} \frac{(u(x+y)-u(x))(v(x+y)-v(x))}{|y|^{d+\alpha}} d y \\
& =\int_{\mathbb{R}^{d}-\{0\}}(u(x+y)-u(x))(v(x+y)-v(x)) \nu(d y), \text { for } u, v \in H^{\frac{\alpha}{2}}\left(\mathbb{R}^{d}\right) .
\end{aligned}
$$

For $u, f, g \in \mathcal{D}(\Delta) \cap L^{\infty}\left(\mathbb{R}^{d}\right)$, one can easily prove the following identity,

$$
\int_{\mathbb{R}^{d}}\langle\nabla f, \nabla g\rangle(x) u(x) d x=-2 \int_{\mathbb{R}^{d}} \frac{1}{2} \Delta f(x) g(x) u(x) d x-\int_{\mathbb{R}^{d}}\langle\nabla f, \nabla u\rangle(x) g(x) d x .
$$

Actually, we can have an identity corresponding to the fractional Laplacian operator similar as identity (3.1).

Lemma 3.1. For $u, f, g \in \mathcal{D}(K) \cap L^{\infty}\left(\mathbb{R}^{d}\right)$, it holds that

$$
\int_{\mathbb{R}^{d}} J(f, g)(x) u(x) d x=-2 \int_{\mathbb{R}^{d}} K f(x) g(x) u(x) d x-\int_{\mathbb{R}^{d}} J(f, u)(x) g(x) d x .
$$

Proof. A direct calculation yields

$$
\begin{aligned}
& \int_{\mathbb{R}^{d}} J(f, g)(x) u(x) d x \\
= & a^{\alpha} C_{d, \alpha} \int_{\mathbb{R}^{d}} \int_{\mathbb{R}^{d}-\{0\}} \frac{[f(x+y)-f(x)][g(x+y)-g(x)]}{|y|^{d+\alpha}} u(x) d y d x \\
= & a^{\alpha} C_{d, \alpha} \int_{\mathbb{R}^{d}} \int_{\mathbb{R}^{d}-\{0\}} \frac{[f(x+y)-f(x)]}{|y|^{d+\alpha}} g(x+y) u(x) d y d x \\
& -a^{\alpha} C_{d, \alpha} \int_{\mathbb{R}^{d}} \int_{\mathbb{R}^{d}-\{0\}} \frac{[f(x+y)-f(x)]}{|y|^{d+\alpha}} g(x) u(x) d y d x \\
= & -a^{\alpha} C_{d, \alpha} \int_{\mathbb{R}^{d}} g(z) d z \int_{\mathbb{R}^{d}-\{0\}} \frac{[f(z+y)-f(z)]}{|y|^{d+\alpha}} u(z+y) d y-\int_{\mathbb{R}^{d}} K f(x) g(x) u(x) d x \\
= & -a^{\alpha} C_{d, \alpha} \int_{\mathbb{R}^{d}} g(z) d z \int_{\mathbb{R}^{d}-\{0\}} \frac{[f(z+y)-f(z)][u(z+y)-u(z)]}{|y|^{d+\alpha}} d y \\
& -a^{\alpha} C_{d, \alpha} \int_{\mathbb{R}^{d}} \int_{\mathbb{R}^{d}-\{0\}} \frac{[f(z+y)-f(z)]}{|y|^{d+\alpha}} g(z) u(z) d y d z-\int_{\mathbb{R}^{d}} K f(x) g(x) u(x) d x \\
= & -\int_{\mathbb{R}^{d}} J(f, u)(z) g(z) d z-2 \int_{\mathbb{R}^{d}} K f(x) g(x) u(x) d x .
\end{aligned}
$$

Corollary 3.2. (1) For $f, g \in \mathcal{D}(\Delta) \cap L^{\infty}\left(\mathbb{R}^{d}\right)$, it follows that $f g \in \mathcal{D}(\Delta)$ in $L^{1}\left(\mathbb{R}^{d}\right)$ and

$$
\Delta(f g)=f \Delta g+g \Delta f+2\langle\nabla f, \nabla g\rangle .
$$

(2) For $f, g \in \mathcal{D}(K) \cap L^{\infty}\left(\mathbb{R}^{d}\right)$, it follows that $f g \in \mathcal{D}(K)$ in $L^{1}\left(\mathbb{R}^{d}\right)$ and

$$
K(f g)=f(K g)+g(K f)+J(f, g) .
$$

(3) For $u \in \mathcal{D}\left(\mathcal{A}^{*}\right) \cap L^{\infty}\left(\mathbb{R}^{d}\right), v \in \mathcal{D}(\mathcal{A}) \cap L^{\infty}$, it follows that $u v \in \mathcal{D}\left(\mathcal{A}^{*}\right)$ in $L^{1}\left(\mathbb{R}^{d}\right)$ and 


$$
\mathcal{A}^{*}(u v)=v\left(\mathcal{A}^{*} u\right)+\left(\frac{1}{2} \Delta v+K v-\langle b, \nabla v\rangle\right) u+J(u, v)+\langle\nabla u, \nabla v\rangle
$$

Proof. We give the proof of (1), then (2) and (3) can be proved similarly.

Set $h:=f \Delta g+g \Delta f+2\langle\nabla f, \nabla g\rangle \in L^{1}\left(\mathbb{R}^{d}\right)$. By identity (3.1), for $u \in C_{c}^{\infty}\left(\mathbb{R}^{d}\right)$,

$$
-\mathcal{E}^{1}(u, f g)=\left(\frac{1}{2} \Delta u, f g\right)=\frac{1}{2}(u, h) .
$$

Let $\left(G_{\lambda}\right)_{\lambda \geq 0}$ be the resolvent of Dirichlet form $\left(\mathcal{E}^{1}, H^{1}\left(\mathbb{R}^{d}\right)\right)$ and $\mathcal{E}_{\lambda}^{1}(\cdot, \cdot)=\mathcal{E}^{1}(\cdot, \cdot)+\lambda(\cdot, \cdot)$. Then by [6], it follows that

$$
(u, f g)=\mathcal{E}_{\lambda}^{1}\left(G_{\lambda} u, f g\right)=\left(G_{\lambda} u, \lambda f g-\frac{1}{2} h\right)=\left(u, G_{\lambda}\left(\lambda f g-\frac{1}{2} h\right)\right) .
$$

Hence, $f g=G_{\lambda}\left(\lambda f g-\frac{1}{2} h\right) \in \mathcal{D}(\Delta)$ in $L^{1}\left(\mathbb{R}^{d}\right)$, and then $\left(\lambda I-\frac{1}{2} \Delta\right) f g=\lambda f g-\frac{1}{2} h$ induces that $\Delta(f g)=h$ in $L^{1}\left(\mathbb{R}^{d}\right)$.

Setting $\mathcal{G}(f, g)(x):=\langle\nabla f(x), \nabla g(x)\rangle+J(f, g)(x)$ and applying the above corollary, we obtain the following lemma.

Lemma 3.3. Fix $o \in \mathbb{R}^{d}$ and set $p_{t}(x):=p(t, o, x)$, then for $u \in \mathcal{D}(\Delta) \cap L^{\infty}\left(\mathbb{R}^{d}\right)$, it holds that

$$
Q_{0, t} u-u=\int_{0}^{t} Q_{0, r}\left(\frac{1}{2} \Delta u+K u-\langle b, \nabla u\rangle+\frac{\mathcal{G}\left(p_{T-r}, u\right)}{p_{T-r}}\right) d r
$$

Proof. Fixing $x \in \mathbb{R}^{d}$, we obtain that

$$
\begin{aligned}
& p_{T}(x) \int_{0}^{t} Q_{0, r}\left(\frac{1}{2} \Delta u+K u-\langle b, \nabla u\rangle\right) d r \\
= & \int_{0}^{t} \int_{\mathbb{R}^{d}} p(T-r, o, y)\left(\frac{1}{2} \Delta u+K u-\langle b, \nabla u\rangle\right)(y) p(r, y, x) d y d r \\
= & -\int_{0}^{t} \int_{\mathbb{R}^{d}} \mathcal{A}^{*} p_{T-r}(y) u(y) p(r, y, x) d y d r+\int_{0}^{t} \int_{\mathbb{R}^{d}} \mathcal{A} p(r, y, x) p(T-r, o, y) u(y) d y d r \\
& -\int_{0}^{t} \int_{\mathbb{R}^{d}}\left\langle\nabla p_{T-r}, \nabla u\right\rangle p(r, y, x) d y d r-\int_{0}^{t} \int_{\mathbb{R}^{d}} J\left(p_{T-r}, u\right) p(r, y, x) d y d r \\
= & \int_{\mathbb{R}^{d}} p(T-t, o, y) u(y) p(t, y, x) d y-p_{T}(x) u(x) \\
& -\int_{0}^{t} \int_{\mathbb{R}^{d}}\left(\left\langle\nabla p_{T-r}, \nabla u\right\rangle+J\left(p_{T-r}, u\right)\right) p(r, y, x) d y d r,
\end{aligned}
$$

where the second equality is derived from Corollary 3.2, and the last equality is obtained by $\mathcal{A}^{*} p(t, o, y)=\partial_{t} p(t, o, y), \mathcal{A} p(t, y, x)=\partial_{t} p(t, y, x)$. Dividing both sides of the above equality by $p_{T}(x)$, then by the definition of semigroup $Q_{0, t}$, the lemma is proved.

Fixing $o \in \mathbb{R}^{d}$, for $u \in \mathcal{D}(\Delta) \cap L^{\infty}\left(\mathbb{R}^{d}\right)$ and $t \in[0, T]$, we define a process as follows,

$$
\begin{aligned}
\left.\bar{M} u\right|_{T-t} ^{T}:=u\left(X_{T-t}\right) & -u\left(X_{T}\right)-\int_{0}^{t}\left(\frac{1}{2} \Delta u+K u-\langle b, \nabla u\rangle\right)\left(X_{T-r}\right) d r \\
& -\int_{0}^{t} \frac{\mathcal{G}\left(p_{T-r}, u\right)\left(X_{T-r}\right)}{p_{T-r}\left(X_{T-r}\right)} d r
\end{aligned}
$$

properties of which are given in the next proposition. 
Proposition 3.4. For any $u \in \mathcal{D}(\Delta) \cap L^{\infty}\left(\mathbb{R}^{d}\right)$, the following two assertions hold.

(1) $\left\{\left.\bar{M} u\right|_{T-t} ^{T}\right\}_{t \in[0, T]}$ is a martingale with respect of the filtration $\mathcal{F}_{t}^{\prime}=\sigma\left\{X_{T-s}, s \in\right.$ $[0, t]\}, t \in[0, T]$, and

$$
\left.\bar{M} u\right|_{t} ^{T}-\left.\bar{M} u\right|_{s} ^{T}=\left.\bar{M} u\right|_{t} ^{s}, \quad \text { for } 0 \leq t \leq s \leq T
$$

(2) It holds that, for $t \in[0, T]$,

$$
u\left(X_{t}\right)-u\left(X_{0}\right)=\left.\frac{1}{2} M u\right|_{0} ^{t}-\frac{1}{2}\left(\left.\bar{M} u\right|_{0} ^{T}-\left.\bar{M} u\right|_{t} ^{T}\right)+\int_{0}^{t}\langle b, \nabla u\rangle\left(X_{r}\right) d r-\frac{1}{2} \int_{0}^{t} \frac{\mathcal{G}\left(p_{r}, u\right)\left(X_{r}\right)}{p_{r}\left(X_{r}\right)} d r .
$$

Proof. This proposition follows Proposition 3.1 in [8]. Hence, we just give the sketch of the proof. The first assertion can be obtained by the Markov property of the reverse process $\left\{X_{T-t}\right\}$ and the expression of the semigroup in Lemma 3.3. The second one is proved as follows: by definition of backward martingale in (3.3), we have

$$
\begin{aligned}
u\left(X_{t}\right)-u\left(X_{0}\right)=\left.\bar{M} u\right|_{t} ^{T}-\left.\bar{M} u\right|_{0} ^{T}-\int_{T-t}^{T}\left(\frac{1}{2} \Delta u+K u-\langle b, \nabla u\rangle\right)\left(X_{T-r}\right) d r \\
\quad-\int_{T-t}^{T} \frac{\mathcal{G}\left(p_{T-r}, u\right)\left(X_{T-r}\right)}{p_{T-r}\left(X_{T-r}\right)} d r \\
=-\left.\bar{M} u\right|_{0} ^{t}-\int_{0}^{t}\left(\frac{1}{2} \Delta u+K u-\langle b, \nabla u\rangle\right)\left(X_{r}\right) d r-\int_{0}^{t} \frac{\mathcal{G}\left(p_{r}, u\right)\left(X_{r}\right)}{p_{r}\left(X_{r}\right)} d r .
\end{aligned}
$$

Recalling the Fukushima decomposition

$$
u\left(X_{t}\right)-u\left(X_{0}\right)=\left.M u\right|_{0} ^{t}+\int_{0}^{t}\left(\frac{1}{2} \Delta u+K u+\langle b, \nabla u\rangle\right)\left(X_{r}\right) d r
$$

and adding the two equations above, we have

$$
2\left(u\left(X_{t}\right)-u\left(X_{0}\right)\right)=\left.M u\right|_{0} ^{t}-\left.\bar{M} u\right|_{0} ^{t}+2 \int_{0}^{t}\langle b, \nabla u\rangle\left(X_{r}\right) d r-\int_{0}^{t} \frac{\mathcal{G}\left(p_{r}, u\right)\left(X_{r}\right)}{p_{r}\left(X_{r}\right)} d r .
$$

Hence, the desired result is obtained.

The following theorem can be obtained by approximating $u \in H^{1}\left(\mathbb{R}^{d}\right)$ with a sequence of $u_{n} \in \mathcal{D}(\Delta)$ in $H^{1}\left(\mathbb{R}^{d}\right)$.

Theorem 3.5. For $u \in H^{1}\left(\mathbb{R}^{d}\right)$, there exists a unique backward martingale $\left\{\left.\bar{M} u\right|_{T-t} ^{T}\right\}_{t \in[0, T]}$ with respect to the backward filtration $\left\{\mathcal{F}_{t}^{\prime}\right\}_{t \in[0, T]}$. Its bracket process is

$$
\left\langle\left.\bar{M} u\right|_{.} ^{T},\left.\bar{M} u\right|_{.} ^{T}\right\rangle_{t}=\int_{t}^{T} \mathcal{G}(u, u)\left(X_{r}\right) d r
$$

Moreover, the following forward-backward martingale decomposition holds,

$$
u\left(X_{t}\right)-u\left(X_{0}\right)=\left.\frac{1}{2} M u\right|_{0} ^{t}-\left.\frac{1}{2} \bar{M} u\right|_{0} ^{t}+\int_{0}^{t}\langle b, \nabla u\rangle\left(X_{r}\right) d r-\frac{1}{2} \int_{0}^{t} \frac{\mathcal{G}\left(p_{r}, u\right)\left(X_{r}\right)}{p_{r}\left(X_{r}\right)} d r .
$$

Remark 3.6. Let $\mu$ be a probability measure on $\mathbb{R}^{d}$, then $\mathbb{P}^{\mu}(\cdot):=\int_{\mathbb{R}^{d}} \mathbb{P}^{x}(\cdot) \mu(d x)$ is a probability measure on $(\Omega, \mathcal{F})$ and the density of semigroup $P_{t}$ under $\mathbb{P}^{\mu}$ is $p_{r}^{\mu}(y)=$ $\int_{\mathbb{R}^{d}} p(r, x, y) \mu(d x)$. The same proof as above implies that Theorem 3.5 also holds under $\mathbb{P}^{\mu}$. 
For any $u \in H^{1}\left(\mathbb{R}^{d}\right)$ and $\alpha \in(0,1)$, since $H^{1}\left(\mathbb{R}^{d}\right) \subset H^{\alpha}\left(\mathbb{R}^{d}\right), u$ lies in the domain of operator $K$. We define

$$
\int_{s}^{t} \nabla u * d X:=\left.M u\right|_{s} ^{t}+\left.\bar{M} u\right|_{s} ^{t}+\int_{s}^{t}\left(2 K u\left(X_{r}\right)+\frac{\mathcal{G}\left(u, p_{r}^{\mu}\right)}{p_{r}^{\mu}}\left(X_{r}\right)\right) d r
$$

which actually does not depend on the initial measure $\mu$. This stochastic $*_{\text {-integral has }}$ an important and helpful property shown in the following lemma.

Lemma 3.7. For $u \in H^{1}\left(\mathbb{R}^{d}\right), \alpha \in(0,1)$ and $s \in[0, T], \int_{s}^{t} \nabla u * d X, t \in[s, T]$ is a zeroenergy functional. Moreover, if $\Delta u=h$ with $h \in L^{2}\left(\mathbb{R}^{d}\right)$ in weak sense, that is, for any $\phi \in C_{c}^{\infty}\left(\mathbb{R}^{d}\right)$,

$$
\int_{\mathbb{R}^{d}}\langle\nabla \phi(x), \nabla u(x)\rangle d x=-\int_{\mathbb{R}^{d}} h(x) \phi(x) d x
$$

then it holds that

$$
\int_{s}^{t} \nabla u * d X=-\int_{s}^{t} h\left(X_{r}\right) d r, \quad \text { for } s<t
$$

Proof. By (3.4) and Fukushima's decomposition (2.5), we find that

$$
\left.\frac{1}{2} M u\right|_{s} ^{t}-\left.\frac{1}{2} \bar{M} u\right|_{s} ^{t}+\int_{s}^{t}\langle b, \nabla u\rangle\left(X_{r}\right) d r-\frac{1}{2} \int_{s}^{t} \frac{\mathcal{G}\left(p_{r}^{\mu}, u\right)\left(X_{r}\right)}{p_{r}^{\mu}\left(X_{r}\right)} d r=\left.M u\right|_{s} ^{t}+\left.N u\right|_{s} ^{t} .
$$

Hence,

$$
\left.M u\right|_{s} ^{t}+\left.\bar{M} u\right|_{s} ^{t}=2 \int_{s}^{t}\langle b, \nabla u\rangle\left(X_{r}\right) d r-\int_{s}^{t} \frac{\mathcal{G}\left(p_{r}^{\mu}, u\right)\left(X_{r}\right)}{p_{r}^{\mu}\left(X_{r}\right)} d r-\left.2 N u\right|_{s} ^{t}
$$

is a zero-energy functional. Hence, $\int_{s}^{t} \nabla u * d X$ is also a zero-energy functional.

For the second assertion, we only need to show that the zero-energy process

$$
\int_{s}^{t} \nabla u * d X+\int_{s}^{t} h\left(X_{r}\right) d r=\left.M u\right|_{s} ^{t}+\left.\bar{M} u\right|_{s} ^{t}+\int_{s}^{t}\left(2 K u\left(X_{r}\right)+\frac{\mathcal{G}\left(u, p_{r}^{\mu}\right)}{p_{r}^{\mu}}\left(X_{r}\right)+h\left(X_{r}\right)\right) d r
$$

is a martingale. Actually, we will prove that, for fixed $t \in(0, T)$, "backward" process

$$
\left.M u\right|_{s} ^{t}+\int_{s}^{t}\left(2 K u\left(X_{r}\right)+\frac{\mathcal{G}\left(u, p_{r}^{\mu}\right)}{p_{r}^{\mu}}\left(X_{r}\right)+h\left(X_{r}\right)\right) d r, \quad s \in[0, t]
$$

is a backward martingale with respect to the backward filtration. Due to the Markovian property of the reversed process $\left\{X_{T-t}\right\}$, we only need to prove, for any $t \in[0, T]$,

$$
E^{\mu}\left[\left.M u\right|_{s} ^{t}+\int_{s}^{t}\left(2 K u\left(X_{r}\right)+\frac{\mathcal{G}\left(u, p_{r}^{\mu}\right)}{p_{r}^{\mu}}\left(X_{r}\right)+h\left(X_{r}\right)\right) d r \mid X_{t}\right]=0 .
$$

For any $\phi \in C_{c}^{\infty}\left(\mathbb{R}^{d}\right)$, set $f(r, x):=P_{t-r} \phi(x)$. Since $\partial_{r} f=-\mathcal{A} f$, it is easy to check that $f\left(r, X_{r}\right)$ is a martingale and

$$
\begin{aligned}
\phi\left(X_{t}\right)=f\left(t, X_{t}\right)= & f\left(s, X_{s}\right)+\sum_{i=1}^{d} \int_{s}^{t} \partial_{i} f\left(r, X_{r}\right) d W_{r}^{i} \\
& +\int_{s}^{t} \int_{\mathbb{R}^{d}}\left(f\left(r, X_{r-}+z\right)-f\left(r, X_{r-}\right)\right) \tilde{N}(d z, d r) .
\end{aligned}
$$


With the help of Itô's formula, we have

$$
\begin{aligned}
& E^{\mu}\left[\left(\left.M u\right|_{s} ^{t}+\int_{s}^{t} 2 K u\left(X_{r}\right)+\frac{\mathcal{G}\left(u, p_{r}^{\mu}\right)}{p_{r}^{\mu}}\left(X_{r}\right)+h\left(X_{r}\right) d r\right) \phi\left(X_{t}\right)\right] \\
&=E^{\mu}\left[\int_{s}^{t} 2 f\left(r, X_{r}\right) K u\left(X_{r}\right)+\frac{\mathcal{G}\left(u, p_{r}^{\mu}\right)}{p_{r}^{\mu}}\left(X_{r}\right) f\left(r, X_{r}\right)+h\left(X_{r}\right) f\left(r, X_{r}\right)\right. \\
&\left.\quad+\sum_{i=1}^{d} \partial_{i} f\left(r, X_{r}\right) \partial_{i} u\left(X_{r}\right) d r\right] \\
&+E^{\mu}\left[\int_{s}^{t}\left(f\left(r, X_{r-}+z\right)-f\left(r, X_{r-}\right)\right)\left(u\left(X_{r-}+z\right)-u\left(X_{r-}\right)\right) \nu(d z) d r\right] \\
&=\int_{s}^{t} \int_{\mathbb{R}^{d}} 2 f(y) K u(y) p_{r}^{\mu}(y)+\mathcal{G}\left(u, p_{r}^{\mu}\right)(y) f(r, y)+h(y) f(r, y) p_{r}^{\mu}(y) \\
& \quad+\langle\nabla f(r, y), \nabla u(y)\rangle p_{r}^{\mu}(y) d y d r \\
&+\int_{s}^{t} \int_{\mathbb{R}^{d}} J(f, u)(y) p_{r}^{\mu}(y) d y d r \\
&=\int_{s}^{t} \int_{\mathbb{R}^{d}} h(y) f(r, y) p_{r}^{\mu}(y)+\left\langle\nabla u(y), \nabla\left(f(r, y) p_{r}^{\mu}(y)\right)\right\rangle d y d r=0,
\end{aligned}
$$

where the last but one equality is obtained by Lemma 3.1. The last equality is deduced by the condition $\Delta u=h$ in weak sense and $f(r, \cdot) p_{r}^{\mu}(\cdot)$ is in the closure of $C_{c}^{\infty}\left(\mathbb{R}^{d}\right)$ for every $r \in[0, T]$.

Hence, we have proved that the process (3.6) is a backward martingale under probability $\mathbb{P}^{\mu}$. Since $\left\{\left.\bar{M} u\right|_{s} ^{t}, s \in[0, t]\right\}$ is another backward martingale, we know that $\int_{s}^{t} \nabla u * d X+\int_{s}^{t} h\left(X_{r}\right) d r$ is a backward martingale with zero energy. Hence, it is a null process.

Combing (2.5), (3.4) and (3.5), we obtain the following proposition, which gives a further description of the zero-energy functional in Fukushima decomposition.

Proposition 3.8. For $u \in H^{1}\left(\mathbb{R}^{d}\right)$ and $\alpha \in(0,1)$, we have the following decomposition

$$
u\left(X_{t}\right)-u\left(X_{s}\right)=\left.M u\right|_{s} ^{t}+\int_{s}^{t} K u\left(X_{r}\right)+\langle b, \nabla u\rangle\left(X_{r}\right) d r-\frac{1}{2} \int_{s}^{t} \nabla u * d X, \quad \text { for } s<t .
$$

Remark 3.9. When a function $u \in H_{l o c}^{1}\left(\mathbb{R}^{d}\right)$ (i.e., for any $\left.\varphi \in C_{c}^{1}\left(\mathbb{R}^{d}\right), u \varphi \in H^{1}\left(\mathbb{R}^{d}\right)\right)$ is considered, we can still define $\left.M u\right|_{0} ^{t},\left.\bar{M} u\right|_{t} ^{T}$, for $t \in[0, T]$, which turn out to be (local) martingale and (local) backward martingale respectively (see $[6,18]$ ). In this case, we are used to denoting by $M^{i}$ and $\bar{M}^{i}$ the local martingale and the local backward martingale which are associated with the coordinate function $u_{i}(x)=x^{i}$, for $i=1, \cdots, d$. Since we consider the time evolution problem in later discussion, we define the stochastic *-integral for function $g \in L^{2}\left([0, T] ; H^{1}\left(\mathbb{R}^{d}\right)\right)$ as follows,

$$
\begin{aligned}
\int_{s}^{t} \nabla g * d X:=\sum_{i=1}^{d}\left(\int_{s}^{t} \partial_{i} g_{r}\left(X_{r}\right) d M_{r}^{i}+\int_{s}^{t} \partial_{i} g_{r}\left(X_{r}\right) \overleftarrow{d \bar{M}_{r}^{i}}\right) \\
+\int_{s}^{t}\left(2 K g_{r}\left(X_{r}\right)+\frac{\mathcal{G}\left(g_{r}, p_{r}^{\mu}\right)}{p_{r}^{\mu}}\left(X_{r}\right)\right) d r
\end{aligned}
$$

Here, the backward stochastic integral is defined as

$$
\int_{s}^{t} \partial_{i} g_{r}\left(X_{r}\right) \overleftarrow{d \bar{M}_{t}^{i}}:=\left.\left(L^{2}-\right) \lim _{\delta \rightarrow 0} \sum_{j=0}^{n-1} \partial_{i} g_{t_{j+1}}\left(X_{t_{j+1}}\right) \bar{M}^{i}\right|_{t_{j}} ^{t_{j+1}}
$$


where the limit is taken over the partition $s=t_{0}<t_{1}<\cdots<t_{n}=t$ and $\delta=\max _{j}\left(t_{j+1}-\right.$ $\left.t_{j}\right)$.

Similarly as the proof of Lemma 3.7 and Lemma 3.1 in [18], we have the following result.

Corollary 3.10. For $g \in L^{2}\left([0, T] ; H^{1}\left(\mathbb{R}^{d}\right)\right), \alpha \in(0,1)$ and $s \in[0, T], \int_{s}^{t} \nabla g * d X, t \in[s, T]$ is a zero-energy functional. Moreover, if $\Delta g=F$ with $F \in L^{2}\left([0, T] ; L^{2}\left(\mathbb{R}^{d}\right)\right)$ in weak sense, i.e., for any $\phi \in \mathcal{D}_{T}$,

$$
\int_{0}^{T} \int_{\mathbb{R}^{d}}\left\langle\nabla g_{r}, \nabla \phi_{r}\right\rangle(x) d x d r=-\int_{0}^{T} \int_{\mathbb{R}^{d}} F_{r}(x) \phi_{r}(x) d x d r,
$$

then it holds that

$$
\int_{s}^{t} \nabla g * d X=-\int_{s}^{t} F_{r}\left(X_{r}\right) d r, \quad \text { for } \quad s<t
$$

\subsection{The case of symmetric Markov process}

In this subsection, we assume that $b=0$. Then we consider a symmetric operator $\mathcal{A}=\frac{1}{2} \Delta+K$ corresponding to a symmetric Markov process $\left\{X_{t}\right\}$.

Let $\Omega^{1}:=\mathcal{C}\left([0, \infty) ; \mathbb{R}^{d}\right)$ be the space of continuous trajectories. The canonical process $\left(W_{t}\right)_{t \geq 0}$ is defined by $W_{t}\left(\omega^{1}\right)=\omega^{1}(t)$, for any $\omega^{1} \in \Omega^{1}, t \geq 0$ and the shift operator, $\theta_{t}^{1}: \Omega^{1} \longrightarrow \Omega^{1}$, is defined by $\theta_{t}^{1}\left(\omega^{1}\right)(s)=\omega^{1}(t+s)$, for any $s, t \geq 0$. The canonical filtration $\mathcal{F}_{t}^{1}=\sigma\left(W_{s} ; s \leq t\right)$ is completed by a standard procedure with respect to the probability measures which are produced by transition function

$$
p_{t}(x, d y)=q_{t}(x-y) d y, \quad t>0, \quad x \in \mathbb{R}^{d},
$$

where $q_{t}(x)=(2 \pi t)^{-\frac{d}{2}} \exp \left(-|x|^{2} / 2 t\right)$ is the Gaussian density. Thus, we get a continuous Hunt process $\left(\Omega^{1}, W_{t}, \theta_{t}^{1}, \mathcal{F}^{1}, \mathcal{F}_{t}^{1}, \mathbb{P}_{1}^{x}\right) . \mathbb{P}_{1}^{0}$ is the Wiener measure, which is supported by the set $\Omega_{0}^{1}=\left\{\omega^{1} \in \Omega^{1}, w^{1}(0)=0\right\}$. We set $\Pi_{0}\left(\omega^{1}\right)(t)=\omega^{1}(t)-\omega^{1}(0), t \geq 0$, which defines a map $\Pi_{0}: \Omega^{1} \rightarrow \Omega_{0}^{1}$. Then $\Pi=\left(W_{0}, \Pi_{0}\right): \Omega^{1} \rightarrow \mathbb{R}^{d} \times \Omega_{0}^{1}$ is a bijection. For each probability measure on $\mathbb{R}^{d}$, the probability $\mathbb{P}^{\mu}$ of the Brownian motion started with the initial distribution $\mu$ is given by

$$
\mathbb{P}_{1}^{\mu}=\Pi^{-1}\left(\mu \otimes \mathbb{P}_{1}^{0}\right)
$$

In particular, for the Lebesgue measure in $\mathbb{R}^{d}$, which we denote by $m=d x$, we have

$$
\mathbb{P}_{1}^{m}=\Pi^{-1}\left(d x \otimes \mathbb{P}_{1}^{0}\right)
$$

Denote $\Omega^{2}:=D\left([0, T] ; \mathbb{R}^{d}\right)$ as the Skorohod space. The canonical processs $\left(V_{t}\right)_{t}$ and the shift operator $\theta_{t}^{2}$ can be defined similarly as $\left(W_{t}\right)_{t}$ and $\theta_{t}^{1}$ given above respectively. Hence, $\left(\Omega^{2}, V_{t}, \theta_{t}^{2}, \mathcal{F}^{2}, \mathcal{F}_{t}^{2}, \mathbb{P}_{2}^{x}\right)$ is a Hunt process corresponding to Dirichlet form $\mathcal{E}^{2}$.

We consider the sample space $\Omega:=\Omega^{1} \times \Omega^{2}$ and the process $\left(X_{t}\right)_{t \geq 0}$ defined by $X_{t}\left(\omega^{1}, \omega^{2}\right)=W_{t}\left(\omega^{1}\right)+V_{t}\left(\omega^{2}\right)$ for $t \geq 0$. The shift operator $\Theta_{t}: \Omega \longrightarrow \Omega$ is defined by $\Theta_{t}\left(w^{1}, w^{2}\right)(s)=\left(w^{1}(t+s), w^{2}(t+s)\right)$, for any $s, t \geq 0$. The $\sigma$-field $\mathcal{F}$ and filtration $\mathcal{F}_{t}$ are given by $\mathcal{F}:=\mathcal{F}^{1} \times \mathcal{F}^{2}$ and $\mathcal{F}_{t}:=\mathcal{F}_{t}^{1} \times \mathcal{F}_{t}^{2}$. The family of probability measures $\left\{\mathbb{P}^{x}\right\}_{x}$ is defined by $\mathbb{P}^{x}:=\mathbb{P}_{1}^{x} \times \mathbb{P}_{2}^{x}$. We see that $\left(\Omega, X_{t}, \Theta_{t}, \mathcal{F}, \mathcal{F}_{t}, \mathbb{P}^{x}\right)$ is a homogeneous Markov process related to the symmetric Dirichlet form $\left(\mathcal{E}, H^{1}\left(\mathbb{R}^{d}\right)\right)$. For the process $X$, we have the following decomposition, for $\alpha \in(0,1)$,

$$
\begin{aligned}
X_{t} & =X_{0}+W_{t}+\int_{0}^{t} \int_{|z| \geq 1} z N(d z, d s)+\int_{0}^{t} \int_{|z|<1} z \tilde{N}(d z, d s) \\
& =X_{0}+W_{t}+\int_{0}^{t} \int_{\mathbb{R}^{d}} z N(d z, d s), \quad t \geq 0 .
\end{aligned}
$$


Since $X$ is symmetric and Lebesgue measure $m$ is invariant, then

$$
p_{r}^{m}(y)=\int_{\mathbb{R}^{d}} p(r, x, y) m(d x)=\int_{\mathbb{R}^{d}} p(r, y, x) m(d x)=1,
$$

which induces that $\mathcal{G}\left(p_{r}^{m}, \phi\right)=0$ for any $\phi \in H^{1}\left(\mathbb{R}^{d}\right)$.

In this symmetric case, for any $u \in H^{1}\left(\mathbb{R}^{d}\right)$, the forward-backward martingale decomposition in Theorem 3.5 is the decomposition proved in [6],

$$
u\left(X_{t}\right)-u\left(X_{0}\right)=\left.\frac{1}{2} M u\right|_{0} ^{t}-\frac{1}{2}\left(\left.\bar{M} u\right|_{0} ^{T}-\left.\bar{M} u\right|_{t} ^{T}\right), \quad t \geq 0 .
$$

For any $u \in H^{1}\left(\mathbb{R}^{d}\right)$ and $\alpha \in(0,1)$, we have

$$
\int_{s}^{t} \nabla u * d X=\left.M u\right|_{s} ^{t}+\left.\bar{M} u\right|_{s} ^{t}+2 \int_{s}^{t} K u\left(X_{r}\right) d r, \quad s<t .
$$

Thus, by Proposition 3.8, we have the following relation,

$$
u\left(X_{t}\right)-u\left(X_{s}\right)=\left.M u\right|_{s} ^{t}+\int_{s}^{t} K u\left(X_{r}\right) d r-\frac{1}{2} \int_{s}^{t} \nabla u * d X, \quad s<t .
$$

In particular, if $u \in \mathcal{D}(\Delta)$, by Lemma 3.7, we have

$$
\int_{s}^{t} \nabla u * d X=-\int_{s}^{t} \Delta u\left(X_{r}\right) d r
$$

In this case, decomposition (3.8) turns out to be

$$
u\left(X_{t}\right)-u\left(X_{s}\right)=\left.M u\right|_{s} ^{t}+\int_{s}^{t}(L+K) u\left(X_{r}\right) d r, \quad s<t .
$$

This equation coincides with the one obtained by applying Itô's formula to $u\left(X_{t}\right)$.

\section{A probabilistic interpretation of stochastic PIDE with diver- gence term}

In this section, applying the forward-backward martingale decomposition obtained in Theorem 3.5, Fukushima decomposition obtained in Proposition 3.8 and letting $u$ be the solution of (1.1), we will give a decomposition of the Dirichlet process $u_{t}\left(X_{t}\right)$ and then the probabilistic interpretation is presented.

Let $B:=\left(B_{t}\right)_{t \geq 0}$ be a standard $d^{1}$-dimensional Brownian motion on a probability space $\left(\Omega^{\prime}, \mathcal{F}^{B}, \mathbb{P}^{\prime}\right)$. Over the time interval $[0, T]$, we define the backward filtration $\left(\mathcal{F}_{s, T}^{B}\right)_{s \in[0, T]}$ where $\mathcal{F}_{s, T}^{B}$ is the completion in $\mathcal{F}^{B}$ of $\sigma\left(B_{r}-B_{s} ; s \leq r \leq T\right)$.

We denote by $\mathcal{H}_{T}$ the space of $H^{1}\left(\mathbb{R}^{d}\right)$-valued $\mathcal{F}_{t, T}^{B}$-predictable processes $\left(u_{t}\right)_{0 \leq t \leq T}$ such that the trajectories $t \rightarrow u_{t}$ are in $\widetilde{F}$ a.s. and $E^{\prime}\|u\|_{T}^{2}<\infty$, where $E^{\prime}$ denotes expectation under probability $\mathbb{P}^{\prime}$.

In the remainder of this paper we assume that final condition $\Phi$ is a given function in $L^{2}\left(\mathbb{R}^{d}\right)$ and coefficients

$$
\begin{aligned}
f & :[0, T] \times \Omega^{\prime} \times \mathbb{R}^{d} \times \mathbb{R} \times \mathbb{R}^{d} \rightarrow \mathbb{R}, \\
g & =\left(g_{1}, \cdots, g_{d}\right):[0, T] \times \Omega^{\prime} \times \mathbb{R}^{d} \times \mathbb{R} \times \mathbb{R}^{d} \rightarrow \mathbb{R}^{d} \\
h & =\left(h_{1}, \ldots, h_{d^{1}}\right):[0, T] \times \Omega^{\prime} \times \mathbb{R}^{d} \times \mathbb{R} \times \mathbb{R}^{d} \rightarrow \mathbb{R}^{d^{1}}
\end{aligned}
$$

are random functions predictable with respect to the backward filtration $\left(\mathcal{F}_{t, T}^{B}\right)_{t \in[0, T]}$. 
We set

$$
f^{0}:=f(\cdot, \cdot, \cdot, 0,0), \quad g^{0}=\left(g_{1}^{0}, \ldots, g_{d}^{0}\right):=g(\cdot, \cdot, \cdot, 0,0), \quad h^{0}=\left(h_{1}^{0}, \ldots, h_{d^{1}}^{0}\right):=h(\cdot, \cdot, \cdot, 0,0)
$$

and assume the following hypotheses:

Assumption (H) There exist non-negative constants $C, \beta$ and $\gamma$ satisfying that

(i) $\left|f\left(t, \omega^{\prime}, x, y, z\right)-f\left(t, \omega^{\prime}, x, y^{\prime}, z^{\prime}\right)\right| \leq C\left(\left|y-y^{\prime}\right|+\left|z-z^{\prime}\right|\right)$;

(ii) $\left(\sum_{j=1}^{d}\left|g^{i}\left(t, \omega^{\prime}, x, y, z\right)-g^{i}\left(t, \omega^{\prime}, x, y^{\prime}, z^{\prime}\right)\right|^{2}\right)^{\frac{1}{2}} \leq C\left|y-y^{\prime}\right|+\gamma\left|z-z^{\prime}\right|$;

(iii) $\left(\sum_{j=1}^{d^{1}}\left|h^{j}\left(t, \omega^{\prime}, x, y, z\right)-h^{j}\left(t, \omega^{\prime}, x, y^{\prime}, z^{\prime}\right)\right|^{2}\right)^{\frac{1}{2}} \leq C\left|y-y^{\prime}\right|+\beta\left|z-z^{\prime}\right|$;

(iv) the contraction property: $2 \gamma+\beta^{2}<1$.

\section{Assumption (HD2)}

$$
E^{\prime}\left(\left\|f^{0}\right\|_{2,2}^{2}+\left\|g^{0}\right\|_{2,2}^{2}+\left\|h^{0}\right\|_{2,2}^{2}\right)<+\infty
$$

Definition 4.1. We say that $u \in \mathcal{H}_{T}$ satisfying $u(T, x)=\Phi(x), d \mathbb{P}^{\prime} \otimes d x-$ a.e. is a weak solution of stochastic PIDE (1.1) associated to $(\Phi, f, h, g)$, if for each $\varphi \in \mathcal{D}_{T}$ and $t \in[0, T]$,

$$
\begin{aligned}
\left(\Phi, \varphi_{T}\right) & -\left(u_{t}, \varphi_{t}\right)=\int_{t}^{T}\left(\left(u_{s}, \partial_{s} \varphi_{s}\right)+\mathcal{E}\left(u_{s}, \varphi_{s}\right)\right) d s-\int_{t}^{T}\left(f_{s}\left(u_{s}, \nabla u_{s}\right), \varphi_{s}\right) d s \\
& +\int_{t}^{T}\left(g_{s}\left(u_{s}, \nabla u_{s}\right), \nabla \varphi_{s}\right) d s-\int_{t}^{T}\left(h_{s}\left(u_{s}, \nabla u_{s}\right), \varphi_{s}\right) \overleftarrow{d B}_{s}, \quad \mathbb{P}^{\prime}-a . s . .
\end{aligned}
$$

\subsection{Existence and uniqueness}

If $|b|$ is bounded, we define $\tilde{f}(t, x, u, \nabla u):=f(t, x, u, \nabla u)+\langle b, \nabla u\rangle$, then by Theorem 8 in [5], it is follows that stochastic PIDE (1.1) admits a unique weak solution and the solution belongs to $\mathcal{H}_{T}$. We now give an analytical proof of the existence and uniqueness of solution for stochastic PIDE (1.1) in the case that drift term $b$ is in Kato class. From this point of view, this section has its independent interest.

Theorem 4.2. Under assumptions $(H)$ and (HD2), there exists a unique weak solution $u \in \mathcal{H}_{T}$ to stochastic PIDE (1.1). Moreover,

$$
E^{\prime}\left[\sup _{0 \leq t \leq T}\left\|u_{t}\right\|^{2}+\int_{0}^{T}\left\|\nabla u_{t}\right\|^{2} d t\right] \leq C E^{\prime}\left[\|\Phi\|^{2}+\left\|f^{0}\right\|_{2,2}^{2}+\left\|g^{0}\right\|_{2,2}^{2}+\left\|h^{0}\right\|_{2,2}^{2}\right],
$$

where the constant $C$ only depends on the structure coefficients of the stochastic PIDE (1.1).

In order to prove Theorem 4.2, we deal with the mild solution to stochastic PIDE firstly, the definition of which is given as follows. Please note that the term involving divergence will be defined later (see Lemma 4.5).

Definition 4.3. We say that $u \in \mathcal{H}_{T}$ is a mild solution to stochastic PIDE (1.1) with terminal condition $u_{T}=\Phi$, if the following equality is verified almost surely, for each $t \in[0, T]$,

$$
\begin{aligned}
u_{t}=P_{T-t} \Phi+\int_{t}^{T} P_{s-t} f_{s}\left(u_{s}, \nabla u_{s}\right) d s & +\int_{t}^{T} P_{s-t} \operatorname{divg} g_{s}\left(u_{s}, \nabla u_{s}\right) d s \\
& +\int_{t}^{T} P_{s-t} h_{s}\left(u_{s}, \nabla u_{s}\right) \overleftarrow{d B}_{s}
\end{aligned}
$$


Lemma 4.4. If $\Phi \in L^{2}\left(\mathbb{R}^{d}\right)$ and $f \in L^{2}\left([0, T] ; \mathbb{R}^{d}\right)$ are given, then

$$
u_{t}=P_{T-t} \Phi+\int_{t}^{T} P_{s-t} f_{s} d s
$$

is a weak solution of the linear equation

$$
d u_{t}+\mathcal{A} u_{t} d t+f_{t} d t=0
$$

with terminal condition $u_{T}=\Phi$. Moreover, $u \in \tilde{F}$ and the following relation is satisfied

$$
\frac{1}{2}\left\|u_{t}\right\|^{2}+\int_{t}^{T} \mathcal{E}\left(u_{s}\right) d s=\frac{1}{2}\|\Phi\|^{2}+\int_{t}^{T}\left(f_{s}, u_{s}\right) d s, \quad \forall t \in[0, T] .
$$

Proof. Firstly, we assume that $\Phi \in \mathcal{D}(\mathcal{A})$ and $f \in \mathcal{D}_{T}$. Then, it is clear that the maps $t \rightarrow P_{T-t} \Phi, t \rightarrow \int_{t}^{T} P_{s-t} f_{s} d s$ belong to $C([0, T], \mathcal{D}(\mathcal{A}))$ and are $L^{2}\left(\mathbb{R}^{d}\right)$-differentiable with continuous derivatives. Then it follows that $t \rightarrow u_{t}$ belongs to $C([0, T], \mathcal{D}(\mathcal{A}))$, and it is also $L^{2}\left(\mathbb{R}^{d}\right)$-differentiable with continuous derivative. Hence, we have

$$
\begin{aligned}
\partial_{t} u_{t} & =\partial_{t} P_{T-t} \Phi+\partial_{t} \int_{t}^{T} P_{s-t} f_{s} d s \\
& =-\mathcal{A} P_{T-t} \Phi-\int_{t}^{T} \mathcal{A} P_{s-t} f_{s} d s-f_{t} \\
& =-\mathcal{A} u_{t}-f_{t} .
\end{aligned}
$$

Applying formula for integration by parts, we obtain that, for all $\varphi \in \mathcal{D}_{T}$,

$$
\int_{t}^{T}\left(u_{s}, \partial_{s} \varphi_{s}\right) d s+\int_{t}^{T} \mathcal{E}\left(u_{s}, \varphi_{s}\right) d s=\left(\Phi, \varphi_{T}\right)-\left(u_{t}, \varphi_{t}\right)+\int_{t}^{T}\left(\varphi_{s}, f_{s}\right) d s
$$

Since we also have that, for all $t \in[0, T]$,

$$
\int_{t}^{T}\left(\partial_{s} u_{s}, u_{s}\right) d s=\|\Phi\|^{2}-\left\|u_{t}\right\|^{2}-\int_{t}^{T}\left(u_{s}, \partial_{s} u_{s}\right) d s
$$

it follows that

$$
\begin{aligned}
\|\Phi\|^{2}-\left\|u_{t}\right\|^{2} & =2 \int_{t}^{T}\left(u_{s}, \partial_{s} u_{s}\right) d s=2 \int_{t}^{T}\left(u_{s},-\mathcal{A} u_{s}-f_{s}\right) d s \\
& =2 \int_{t}^{T} \mathcal{E}\left(u_{s}\right) d s-2 \int_{t}^{T}\left(u_{s}, f_{s}\right) d s
\end{aligned}
$$

which proves the equality (4.4). Combing with the form of $\mathcal{E}$, we get

$$
\left\|u_{t}\right\|^{2}+2 \int_{t}^{T}\left(\mathcal{E}^{1}\left(u_{s}\right)+\mathcal{E}^{2}\left(u_{s}\right)\right) d s=\|\Phi\|^{2}+2 \int_{t}^{T} \int_{\mathbb{R}^{d}}\left\langle b, \nabla u_{s}\right\rangle(x) u_{s}(x) d x d s+2 \int_{t}^{T}\left(u_{s}, f_{s}\right) d s .
$$

By applying Hölder's inequality, Kato-type inequality (2.1) and Cauchy-Schwartz's inequality, we have, for any $0<\epsilon<1, \epsilon^{\prime}>0$,

$$
\begin{aligned}
& 2 \int_{t}^{T} \int_{\mathbb{R}^{d}}\left\langle b, \nabla u_{s}\right\rangle(x) u_{s}(x) d x d s \\
\leq & 2 \int_{t}^{T}\left(\int_{\mathbb{R}^{d}}\left|\nabla u_{s}(x)\right|^{2} d x\right)^{\frac{1}{2}} \cdot\left(\epsilon \int_{\mathbb{R}^{d}}\left|\nabla u_{s}(x)\right|^{2} d x+C_{\epsilon} \int_{\mathbb{R}^{d}}\left|u_{s}(x)\right|^{2} d x\right)^{\frac{1}{2}} d s \\
\leq & \left(\epsilon^{\prime}+\frac{\epsilon}{\epsilon^{\prime}}\right) \int_{t}^{T} \int_{\mathbb{R}^{d}}\left|\nabla u_{s}(x)\right|^{2} d x d s+\frac{C_{\epsilon}}{\epsilon^{\prime}} \int_{t}^{T} \int_{\mathbb{R}^{d}}\left|u_{s}(x)\right|^{2} d x d s
\end{aligned}
$$


and

$$
2 \int_{t}^{T}\left(u_{s}, f_{s}\right) d s \leq \int_{t}^{T}\left\|u_{s}\right\|^{2} d s+\int_{t}^{T}\left\|f_{s}\right\|^{2} d s
$$

Then

$$
\left\|u_{t}\right\|^{2}+\left(1-\epsilon^{\prime}-\frac{\epsilon}{\epsilon^{\prime}}\right) \int_{t}^{T}\left\|\nabla u_{s}\right\|^{2} d s \leq\|\Phi\|^{2}+\frac{C_{\epsilon}}{\epsilon^{\prime}} \int_{t}^{T}\left\|u_{s}\right\|^{2} d s+\int_{t}^{T}\left\|f_{s}\right\|^{2} d s .
$$

Taking $\epsilon$ and $\epsilon^{\prime}$ small enough such that $\left(1-\epsilon^{\prime}-\frac{\epsilon}{\epsilon^{\prime}}\right)>0$, and applying Gronwall's inequality, we obtain that

$$
\left\|u_{t}\right\|^{2}+\int_{t}^{T}\left\|\nabla u_{s}\right\|^{2} d s \leq C\left(\|\Phi\|^{2}+\int_{t}^{T}\left\|f_{s}\right\|^{2} d s\right) .
$$

Taking the supremum of the above inequality, we get $\sup _{t \in[0, T]}\left\|u_{t}\right\|^{2}+\int_{0}^{T}\left\|\nabla u_{s}\right\|^{2} d s<\infty$, which implies that $u \in \tilde{F}$. Finally, one can obtain the result in the general case by approximation.

To treat the divergence term, we need to give a precise definition of the integral $\int_{t}^{T} P_{s-t} \operatorname{div} g_{s} d s$, which is just a formal writing with $g \in L^{2}\left([0, T], \mathbb{R}^{d}\right)$. Obviously, this integral is well defined if $g \in \mathcal{D}_{T}$. We therefore define a operator $U: \mathcal{D}_{T} \rightarrow \tilde{F}$ by

$$
(U g)_{t}:=\int_{t}^{T} P_{s-t} \operatorname{div} g_{s} d s, \quad t \in[0, T] .
$$

The next lemma proves that we can extend it by continuity. In the following discussion, we will use the formal expression $\int_{t}^{T} P_{s-t} \operatorname{div} g_{s} d s$ rather than $U g$.

Lemma 4.5. The operator $U$ admits a uniquely determined continuous extension

$$
U: L^{2}\left([0, T], \mathbb{R}^{d}\right) \rightarrow \tilde{F} .
$$

If $g \in L^{2}\left([0, T], \mathbb{R}^{d}\right)$ is given, then $u=U g$ is a weak solution of the linear equation

$$
d u_{t}+\mathcal{A} u_{t} d t+d i v g_{t} d t=0, \quad u_{T}=0
$$

Moreover, the following relation is satisfied

$$
\frac{1}{2}\left\|u_{t}\right\|^{2}+\int_{t}^{T} \mathcal{E}\left(u_{s}\right) d s+\int_{t}^{T}\left(g_{s}, \nabla u_{s}\right) d s=0, \quad \forall t \in[0, T] .
$$

Proof. Assume that $g \in \mathcal{D}_{T}$ firstly, then it is obvious that $\operatorname{div} g_{t} \in \mathcal{D}(\mathcal{A})$. By Lemma 4.4, we deduce that $u=U g$ is a weak solution of (4.5). By (4.4) we get

$$
\left\|(U g)_{t}\right\|^{2}+2 \int_{t}^{T} \mathcal{E}\left((U g)_{s}\right) d s=2 \int_{t}^{T}\left(\operatorname{div} g_{s},(U g)_{s}\right) d s=-2 \int_{t}^{T}\left(g_{s}, \nabla(U g)_{s}\right) d s,
$$

which yields equation (4.6) in this smooth case.

Using Young's and Kato-type inequality, by the similar method in proof of Lemma 4.4, we have

$$
\left\|(U g)_{t}\right\|^{2}+\left(1-\epsilon^{\prime}-\frac{\epsilon}{\epsilon^{\prime}}-\epsilon^{\prime \prime}\right) \int_{t}^{T}\left\|\nabla(U g)_{s}\right\|^{2} d s \leq \frac{C_{\epsilon}}{\epsilon^{\prime}} \int_{t}^{T}\left\|(U g)_{s}\right\|^{2} d s+C_{\epsilon^{\prime \prime}} \int_{t}^{T}\left\|g_{s}\right\|^{2} d s .
$$

We choose $\epsilon, \epsilon^{\prime}$ and $\epsilon^{\prime \prime}$ small enough such that $\left(1-\epsilon^{\prime}-\frac{\epsilon}{\epsilon^{\prime}}-\epsilon^{\prime \prime}\right)>0$. Thanks to Gronwall's lemma, we obtain

$$
\left\|(U g)_{t}\right\|^{2}+\int_{t}^{T}\left\|\nabla(U g)_{s}\right\|^{2} d s \leq C \int_{t}^{T}\left\|g_{s}\right\|^{2} d s .
$$


Then taking the supremum of the above inequality, we get $\|U g\|_{T} \leq C \int_{0}^{T}\left\|g_{s}\right\|^{2} d s$, which implies $U g \in \tilde{F}$ and the continuity of operator $U$. The result with general $g \in L^{2}\left([0, T], \mathbb{R}^{d}\right)$ follows by approximation.

Lemma 4.6. (stochastic term) Let $h \in L^{2}\left(\Omega^{\prime} \times[0, T] \times \mathbb{R}^{d}\right)$ be adapted. Then the process $\beta: t \in[0, T] \rightarrow \int_{t}^{T} P_{s-t} h_{s} \overleftarrow{d B}_{s}$ admits a version in $\mathcal{H}_{T}$, and for any $\varphi \in \mathcal{D}_{T}, t \in[0, T]$, we have

$$
\int_{t}^{T}\left(\beta_{s}, \partial_{s} \varphi_{s}\right) d s+\int_{t}^{T} \mathcal{E}\left(\beta_{s}, \varphi_{s}\right) d s=-\left(\beta_{t}, \varphi_{t}\right)+\int_{t}^{T}\left(h_{s}, \varphi_{s}\right) \overleftarrow{d B}_{s}, \mathbb{P}^{\prime}-a . s .
$$

Proof. Assume first that $h \in L^{2}\left(\Omega^{\prime}\right) \otimes C^{1}([0, T]) \otimes \mathcal{D}(\mathcal{A})$ is adapted. It is clear that the process

$$
\forall t \in[0, T], \beta_{t}=\int_{t}^{T} P_{s-t} h_{s} \overleftarrow{d B}_{s}
$$

is a square integrable $\mathcal{D}(\mathcal{A})$-valued martingale w.r.t the filtration $\left(\mathcal{F}_{t, T}^{B}\right)_{t \in[0, T]}$ (see Proposition 2.3 in [4]). Hence, for all $t \in[0, T]$, we have (see Theorem 3.3 in [17])

$$
\begin{aligned}
\beta_{t} & =\int_{t}^{T} P_{s-s} h_{s} \overleftarrow{d B}_{s}+\int_{t}^{T}\left(\int_{s}^{T} \mathcal{A} P_{u-s} h_{u} \overleftarrow{d B}_{u}\right) d s \\
& =\int_{t}^{T} h_{s} \overleftarrow{d B}_{s}+\int_{t}^{T} \mathcal{A} \beta_{s} d s, \mathbb{P}^{\prime}-\text { a.s. }
\end{aligned}
$$

Thanks to Itô's formula, we have that, for any $\varphi \in \mathcal{D}_{T}, t \in[0, T]$,

$$
-\left(\beta_{t}, \varphi_{t}\right)=\int_{t}^{T}\left(\beta_{s}, \partial_{s} \varphi_{s}\right) d s-\int_{t}^{T}\left(h_{s}, \varphi_{s}\right) \overleftarrow{d B}_{s}-\int_{t}^{T}\left(\mathcal{A} \beta_{s}, \varphi_{s}\right) d s, \mathbb{P}^{\prime}-a . s .
$$

which yields (4.8).

We apply Itô's formula to $\beta^{2}$ and obtain

$$
\left\|\beta_{t}\right\|^{2}+2 \int_{t}^{T} \mathcal{E}\left(\beta_{s}\right) d s=2 \int_{t}^{T}\left(\beta_{s}, h_{s}\right) \overleftarrow{d B}_{s}+\int_{t}^{T}\left\|h_{s}\right\|^{2} d s, \quad \forall t \in[0, T], \quad \mathbb{P}^{\prime}-a . s .
$$

Applying Kato-type inequality, Burkholder-Davies-Gundy's inequality and Gronwall's inequality, we prove $\beta \in \mathcal{H}_{T}$. By the density argument, we get assertions in general case.

The following proposition can be obtained by a similar argument as Proposition 4.9 in [4] or Proposition 7 in [5]. We therefore omit the proof here.

Proposition 4.7. $u \in \mathcal{H}_{T}$ is a weak solution of SPDE (1.1) satisfying (4.1) if and only if $u$ is a mild solution satisfying (4.3).

Now we prove the existence and uniqueness of solution to stochastic PDE (1.1).

Proof of Theorem 4.2: Let $\theta$ and $\delta$ be two positive numbers, which will be determined later. We introduce a norm of space $L^{2}\left(\Omega^{\prime} \times[0, T] ; H^{1}\left(\mathbb{R}^{d}\right)\right)$,

$$
\|u\|_{\theta, \delta}^{2}:=E^{\prime} \int_{0}^{T} e^{\theta s}\left(\delta\left\|u_{s}\right\|^{2}+\left\|\nabla u_{s}\right\|^{2}\right) d s
$$

which is an equivalent norm on $L^{2}\left(\Omega^{\prime} \times[0, T] ; H^{1}\left(\mathbb{R}^{d}\right)\right)$. Consider a mapping $\Lambda: \mathcal{H}_{T} \rightarrow \mathcal{H}_{T}$ defined as follows, 


$$
\begin{aligned}
(\Lambda u)_{t}:= & P_{T-t} \Phi+\int_{t}^{T} P_{s-t} f_{s}\left(u_{s}, \nabla u_{s}\right) d s+\int_{t}^{T} P_{s-t} \operatorname{div} g_{s}\left(u_{s}, \nabla u_{s}\right) d s \\
& +\int_{t}^{T} P_{s-t} h_{s}\left(u_{s}, \nabla u_{s}\right) \overleftarrow{d B}_{s}, \quad \forall u \in \mathcal{H}_{T} .
\end{aligned}
$$

Choosing $u, v \in \mathcal{H}_{T}$, by Itô's formula, we have almost surely,

$$
\begin{aligned}
& \left\|\Lambda u_{0}-\Lambda v_{0}\right\|^{2}+2 \int_{0}^{T} e^{\theta s}\left(\mathcal{E}^{1}\left(\Lambda u_{s}-\Lambda v_{s}\right)+\mathcal{E}^{2}\left(\Lambda u_{s}-\Lambda v_{s}\right)\right) d s \\
= & 2 \int_{0}^{T} \int_{\mathbb{R}^{d}} e^{\theta s}\left\langle b, \nabla\left(\Lambda u_{s}-\Lambda v_{s}\right)\right\rangle(x)\left(\Lambda u_{s}(x)-\Lambda v_{s}(x)\right) d x d s \\
& -\theta \int_{0}^{T} e^{\theta s}\left\|\Lambda u_{s}-\Lambda v_{s}\right\|^{2} d s+2 \int_{0}^{T} e^{\theta s}\left(\Lambda u_{s}-\Lambda v_{s}, f_{s}\left(u_{s}, \nabla u_{s}\right)-f_{s}\left(v_{s}, \nabla v_{s}\right)\right) d s \\
& -2 \int_{0}^{T} \int_{\mathbb{R}^{d}} e^{\theta s}\left\langle\nabla\left(\Lambda u_{s}-\Lambda v_{s}\right), g_{s}\left(u_{s}, \nabla u_{s}\right)-g_{s}\left(v_{s}, \nabla v_{s}\right)\right\rangle(x) d x d s \\
& +2 \int_{0}^{T} e^{\theta s}\left(\Lambda u_{s}-\Lambda v_{s}, h_{s}\left(u_{s}, \nabla u_{s}\right)-h_{s}\left(v_{s}, \nabla v_{s}\right)\right) \overleftarrow{d B} \overleftarrow{s}^{T} \\
& +\int_{0}^{\theta s}\left\|h_{s}\left(u_{s}, \nabla u_{s}\right)-h_{s}\left(v_{s}, \nabla v_{s}\right)\right\|^{2} d s .
\end{aligned}
$$

By Lipschitz condition (H) and some elementary inequalities, we get

$$
\begin{aligned}
& 2 \int_{0}^{T} e^{\theta s}\left(\Lambda u_{s}-\Lambda v_{s}, f_{s}\left(u_{s}, \nabla u_{s}\right)-f_{s}\left(v_{s}, \nabla v_{s}\right) d s\right. \\
\leq & \frac{1}{\epsilon} \int_{0}^{T} e^{\theta s}\left\|\Lambda u_{s}-\Lambda v_{s}\right\|^{2} d s+C \epsilon \int_{0}^{T} e^{\theta s}\left\|u_{s}-v_{s}\right\|^{2} d s+C \epsilon \int_{0}^{T} e^{\theta s}\left\|\nabla\left(u_{s}-v_{s}\right)\right\|^{2} d s
\end{aligned}
$$

and

and

$$
\begin{aligned}
& 2 \int_{0}^{T} \int_{\mathbb{R}^{d}} e^{\theta s}\left\langle\nabla\left(\Lambda u_{s}-\Lambda v_{s}\right), g_{s}\left(u_{s}, \nabla u_{s}\right)-g_{s}\left(v_{s}, \nabla v_{s}\right)\right\rangle(x) d x d s \\
\leq & 2 \int_{0}^{T} e^{\theta s}\left(C\left\|u_{s}-v_{s}\right\|+\gamma\left\|\nabla\left(u_{s}-v_{s}\right)\right\|\right)\left\|\nabla\left(\Lambda u_{s}-\Lambda v_{s}\right)\right\| d s \\
\leq & C \epsilon \int_{0}^{T} e^{\theta s}\left\|\nabla\left(\Lambda u_{s}-\Lambda v_{s}\right)\right\|^{2} d s+\frac{C}{\epsilon} \int_{0}^{T} e^{\theta s}\left\|u_{s}-v_{s}\right\|^{2} d s \\
& +\gamma \int_{0}^{T} e^{\theta s}\left\|\nabla\left(\Lambda u_{s}-\Lambda v_{s}\right)\right\|^{2} d s+\gamma \int_{0}^{T} e^{\theta s}\left\|\nabla\left(u_{s}-v_{s}\right)\right\|^{2} d s
\end{aligned}
$$

$$
\begin{aligned}
& \int_{0}^{T} e^{\theta s}\left\|h_{s}\left(u_{s}, \nabla u_{s}\right)-h_{s}\left(v_{s}, \nabla v_{s}\right)\right\|^{2} d s \\
\leq & C\left(1+\frac{1}{\epsilon}\right) \int_{0}^{T} e^{\theta s}\left\|u_{s}-v_{s}\right\|^{2} d s+\beta^{2}(1+\epsilon) \int_{0}^{T} e^{\theta s}\left\|\nabla\left(u_{s}-v_{s}\right)\right\|^{2} d s
\end{aligned}
$$

and

$$
\begin{aligned}
& 2 \int_{0}^{T} \int_{\mathbb{R}^{d}} e^{\theta s}\left\langle b, \nabla\left(\Lambda u_{s}-\Lambda v_{s}\right)\right\rangle(x)\left(\Lambda u_{s}(x)-\Lambda v_{s}(x)\right) d x d s \\
\leq & 2 \int_{0}^{T}\left(\int_{\mathbb{R}^{d}} e^{\theta s}\left|\nabla\left(\Lambda u_{s}-\Lambda v_{s}\right)\right|^{2} d x\right)^{\frac{1}{2}} \\
& \times\left(\epsilon \int_{\mathbb{R}^{d}} e^{\theta s}\left|\nabla\left(\Lambda u_{s}-\Lambda v_{s}\right)\right|^{2} d x+C_{\epsilon} \int_{\mathbb{R}^{d}} e^{\theta s}\left|\Lambda u_{s}-\Lambda v_{s}\right|^{2} d x\right)^{\frac{1}{2}} d s \\
\leq & \left(\epsilon^{\prime}+\frac{\epsilon}{\epsilon^{\prime}}\right) \int_{0}^{T} \int_{\mathbb{R}^{d}} e^{\theta s}\left|\nabla\left(\Lambda u_{s}-\Lambda v_{s}\right)\right|^{2} d x d s+\frac{C_{\epsilon}}{\epsilon^{\prime}} \int_{0}^{T} \int_{\mathbb{R}^{d}} e^{\theta s}\left|\Lambda u_{s}-\Lambda v_{s}\right|^{2} d x d s .
\end{aligned}
$$


Therefore, it follows that

$$
\begin{aligned}
& \left(\theta-\frac{1}{\epsilon}-\frac{C_{\epsilon}}{\epsilon^{\prime}}\right) E \int_{0}^{T} e^{\theta s}\left\|\Lambda u_{s}-\Lambda v_{s}\right\|^{2} d s+\left(1-C \epsilon-\gamma-\epsilon^{\prime}-\frac{\epsilon}{\epsilon^{\prime}}\right) E \int_{0}^{T} e^{\theta s}\left\|\nabla\left(\Lambda u_{s}-\Lambda v_{s}\right)\right\|^{2} d s \\
& \leq C\left(1+\epsilon+\frac{2}{\epsilon}\right) E \int_{0}^{T} e^{\theta s}\left\|u_{s}-v_{s}\right\|^{2} d s+\left(C \epsilon+\gamma+\beta^{2}(1+\epsilon)\right) E \int_{0}^{T} e^{\theta s}\left\|\nabla\left(u_{s}-v_{s}\right)\right\|^{2} d s .
\end{aligned}
$$

Since $2 \gamma+\beta^{2}<1$, we can choose $\epsilon$ and $\epsilon^{\prime}$ small enough such that

$$
C \epsilon+\gamma+\beta^{2}(1+\epsilon)<1-C \epsilon-\gamma-\epsilon^{\prime}-\frac{\epsilon}{\epsilon^{\prime}},
$$

and then choose $\theta$ satisfying that

$$
\frac{\theta-\frac{1}{\epsilon}-\frac{C_{\epsilon}}{\epsilon^{\prime}}}{1-C \epsilon-\gamma-\epsilon^{\prime}-\frac{\epsilon}{\epsilon^{\prime}}}=\frac{C\left(1+\epsilon+\frac{2}{\epsilon}\right)}{C \epsilon+\gamma+\beta^{2}(1+\epsilon)} .
$$

By setting $\delta=\frac{\theta-\frac{1}{\epsilon}-\frac{C_{\epsilon}}{\epsilon^{\prime}}}{1-C \epsilon-\gamma-\epsilon^{\prime}-\frac{\epsilon}{\epsilon^{\prime}}}$, we find

$$
\forall u, v \in \mathcal{H}_{T}, \quad\|\Lambda(u)-\Lambda(v)\|_{\theta, \delta} \leq \frac{C \epsilon+\gamma+\beta^{2}(1+\epsilon)}{1-C \epsilon-\gamma-\epsilon^{\prime}-\frac{\epsilon}{\epsilon^{\prime}}}\|u-v\|_{\theta, \delta} .
$$

We therefor conclude the result by fixed point theorem.

\subsection{Probabilistic interpretation}

By Theorem 4.2, we know that there is a unique function $u$ satisfying stochastic PIDE (1.1). We then give a probabilistic interpretation for the Dirichlet process $u_{t}\left(X_{t}\right)$ in this section. Please note that we always assume that $\alpha \in(0,1)$ in the following discussion.

In the case that $g$ is independent of $u$ and $\nabla u$, by assumption (HD2), for any $\varphi \in \mathcal{D}_{T}$, we have

$$
\int_{0}^{T}\left(g_{s}, \nabla \varphi_{s}\right) d s \leq\|g\|_{2,2} \cdot\|\nabla \varphi\|_{2,2} \leq\|g\|_{2,2} \cdot\left(\int_{0}^{T}\left\|\varphi_{s}\right\|_{H^{1}\left(\mathbb{R}^{d}\right)}^{2} d s\right)^{\frac{1}{2}} .
$$

By Riesz's representation theorem on Hilbert space $L^{2}\left(\Omega^{\prime} \times[0, T] ; H^{1}\left(\mathbb{R}^{d}\right)\right)$, there exists a unique function $G \in L^{2}\left(\Omega^{\prime} \times[0, T] ; H^{1}\left(\mathbb{R}^{d}\right)\right)$ such that

$$
\int_{0}^{T}\left(g_{s}, \nabla \varphi_{s}\right) d s=\int_{0}^{T}\left(G_{s}, \varphi_{s}\right)_{H^{1}\left(\mathbb{R}^{d}\right)} d s=\int_{0}^{T}\left(G_{s}, \varphi_{s}\right)+\left(\nabla G_{s}, \nabla \varphi_{s}\right) d s, \quad \varphi \in \mathcal{D}_{T} .
$$

Therefore, the weak solution $u$ of stochastic PIDE (1.1) with linear $g=g_{t}(x)$ also satisfies the following equation

$$
\left\{\begin{aligned}
d u_{t}(x)+\left[\mathcal{A} u_{t}(x)+\right. & \left.f_{t}\left(x, u_{t}(x), \nabla u_{t}(x)\right)\right] d t+h_{t}\left(x, u_{t}(x), \nabla u_{t}(x)\right) \cdot \overleftarrow{d B}_{t} \\
& +\left[\Delta G_{t}(x)-G_{t}(x)\right] d t=0, \quad(t, x) \in[0, T] \times \mathbb{R}^{d} \\
u_{T}(x)=\Phi(x), \quad x \in & \mathbb{R}^{d} .
\end{aligned}\right.
$$

Due to the existence and uniqueness of the function $G \in L^{2}\left(\Omega^{\prime} \times[0, T] ; H^{1}\left(\mathbb{R}^{d}\right)\right)$, we define the following process,

$$
\int_{s}^{t} g_{r}\left(X_{r}\right) * d X:=\int_{s}^{t} \nabla G * d X+\int_{s}^{t} G_{r}\left(X_{r}\right) d r, \quad \mathbb{P}^{\prime} \otimes \mathbb{P}-\text { a.s. }
$$


which is a zero-energy process by Corollary 3.10.

A probabilistic interpretation of the solution for SPDE (1.1) is given in the following proposition in the linear situation (i.e., $g$ only depends on $(t, x)$ ). We omit the variables $\omega^{\prime}, \omega$ in the following discussion for simplicity. For example, we denote $u_{t}\left(\omega^{\prime}, X_{t}(\omega)\right)$ by $u_{t}\left(X_{t}\right)$.

Proposition 4.8. Suppose $u \in \mathcal{H}_{T}$ is the weak solution of stochastic PIDE (1.1) with $g$ only depending on $(t, x) \in[0, T] \times \mathbb{R}^{d}$, then the process $u_{t}\left(X_{t}\right)$ admits the following decomposition: for $0<t<T$,

$$
\begin{aligned}
u_{t}\left(X_{t}\right)= & \Phi\left(X_{T}\right)-\left.M u\right|_{t} ^{T}+\int_{t}^{T} f_{r}\left(X_{r}, u_{r}\left(X_{r}\right), \nabla u_{r}\left(X_{r}\right)\right) d r \\
& +\int_{t}^{T} h_{r}\left(X_{r}, u_{r}\left(X_{r}\right), \nabla u_{r}\left(X_{r}\right)\right) \overleftarrow{d B}_{r}-\int_{t}^{T} g_{r}\left(X_{r}\right) * d X, \quad \mathbb{P}^{\prime} \otimes \mathbb{P}-\text { a.s.. }
\end{aligned}
$$

Proof. We consider a sequence $\left(G^{n}\right)_{n \in \mathbb{N}^{*}}$ of smooth functions that approximates $G \in$ $L^{2}\left(\Omega^{\prime} \times[0, T] ; H^{1}\left(\mathbb{R}^{d}\right)\right)$ obtained in (4.11). Let $u^{n}$ be the solution of (4.12) corresponding to $G^{n}$. Then it is known that

$$
\begin{aligned}
u_{t}^{n}\left(X_{t}\right)= & \Phi\left(X_{T}\right)-\left.M u^{n}\right|_{t} ^{T}+\int_{t}^{T} f_{r}\left(X_{r}, u_{r}^{n}\left(X_{r}\right), \nabla u_{r}^{n}\left(X_{r}\right)\right)-G_{r}^{n}\left(X_{r}\right) d r \\
& +\int_{t}^{T} h_{r}\left(X_{r}, u_{r}^{n}\left(X_{r}\right), \nabla u_{r}^{n}\left(X_{r}\right)\right) \overleftarrow{d B}_{r}+\int_{t}^{T} \Delta G_{r}^{n}\left(X_{r}\right) d r, \quad \mathbb{P}^{\prime} \otimes \mathbb{P}-a . s .
\end{aligned}
$$

Since $G^{n}$ is smooth, we have $\int_{s}^{t} \Delta G_{r}^{n}\left(X_{r}\right) d r=-\int_{s}^{t} \nabla G^{n} * d X$ by Corollary 3.10. Then (4.14) can be written as follows

$$
\begin{aligned}
u_{t}^{n}\left(X_{t}\right)= & \Phi\left(X_{T}\right)-\left.M u^{n}\right|_{t} ^{T}+\int_{t}^{T}\left(f_{r}\left(X_{r}, u_{r}^{n}\left(X_{r}\right), \nabla u_{r}^{n}\left(X_{r}\right)\right)-G_{r}^{n}\left(X_{r}\right)\right) d r \\
& +\int_{t}^{T} h_{r}\left(X_{r}, u_{r}^{n}\left(X_{r}\right), \nabla u_{r}^{n}\left(X_{r}\right)\right) \overleftarrow{d B}_{r}-\int_{t}^{T} \nabla G^{n} * d X, \quad \mathbb{P}^{\prime} \otimes \mathbb{P}-\text { a.s.. }
\end{aligned}
$$

By estimate in (4.2), we know that $\left(u^{n}\right)_{n}$ converges strongly to $u$ in $L^{2}\left(\Omega^{\prime} \times[0, T] ; H^{1}\left(\mathbb{R}^{d}\right)\right)$. Passing limits on both sides of (4.15), we obtain

$$
\begin{aligned}
u_{t}\left(X_{t}\right)= & u_{T}\left(X_{T}\right)-\left.M u\right|_{t} ^{T}+\int_{t}^{T}\left(f_{r}\left(X_{r}, u_{r}\left(X_{r}\right), \nabla u_{r}\left(X_{r}\right)\right)-G_{r}\left(X_{r}\right)\right) d r \\
& +\int_{t}^{T} h_{r}\left(X_{r}, u_{r}\left(X_{r}\right), \nabla u_{r}\left(X_{r}\right)\right) \overleftarrow{d B}_{r}-\int_{t}^{T} \nabla G * d X, \quad \mathbb{P}^{\prime} \otimes \mathbb{P}-\text { a.s.. }
\end{aligned}
$$

Therefore, by the definition of $\int_{s}^{t} g * d X$, we prove the desired result.

Taking the conditional expectation on both sides of (4.16) with respect to filtration $\left\{\mathcal{F}_{t}\right\}$, by the Markovian property of $\left\{X_{T-t}\right\}$ and the independence between $\left\{\mathcal{F}_{t}^{B}\right\}$ and $\left\{\mathcal{F}_{t}\right\}$, we obtain the following representation similar to the Feynman-Kac formula.

Corollary 4.9. Let $u \in \mathcal{H}_{T}$ be the solution of stochastic PIDE (1.1), then it holds $\mathbb{P}^{\prime}$ almost surely that

$$
u_{t}(x)=E^{x}\left[\Phi\left(X_{T-t}\right)+\int_{t}^{T} f_{r}\left(X_{r-t}\right) d r-\int_{t}^{T} g_{r}\left(X_{r-t}\right) * d X\right] .
$$

In the general case that $g$ depends on $(t, x, u, \nabla u)$, we set $\tilde{g}(t, x):=g\left(t, x, u_{t}(x), \nabla u_{t}(x)\right)$, then $\tilde{g}(t, x) \in L^{2}\left(\Omega^{\prime} \times[0, T] ; \mathbb{R}^{d}\right)$. Thus, we define, $\mathbb{P}^{\prime} \otimes \mathbb{P}-$ a.s., for $0<s<t<T$,

$$
\int_{s}^{t} g_{r}\left(X_{r}, u_{r}\left(X_{r}\right), \nabla u_{r}\left(X_{r}\right)\right) * d X=\int_{s}^{t} \tilde{g}_{r}\left(X_{r}\right) * d X
$$


Hence, the following theorem is obtained.

Theorem 4.10. Let $u \in \mathcal{H}_{T}$ be the solution of stochastic PIDE (1.1). For any $0<t<T$, the process $u_{t}\left(X_{t}\right)$ admits the following stochastic representation, $\mathbb{P}^{\prime} \otimes \mathbb{P}-$ a.s.,

$$
\begin{aligned}
u_{t}\left(X_{t}\right)= & \Phi\left(X_{T}\right)-\left.M u\right|_{t} ^{T}+\int_{t}^{T} f_{r}\left(X_{r}, u_{r}\left(X_{r}\right), \nabla u_{r}\left(X_{r}\right)\right) d r \\
& +\int_{t}^{T} h_{r}\left(X_{r}, u_{r}\left(X_{r}\right), \nabla u_{r}\left(X_{r}\right)\right) \overleftarrow{d B}_{r}-\int_{t}^{T} g_{r}\left(X_{r}, u_{r}\left(X_{r}\right), \nabla u_{r}\left(X_{r}\right)\right) * d X
\end{aligned}
$$

\section{Backward doubly stochastic differential equations}

In this section we will reveal a connection between the solutions to stochastic PIDEs and a class of BDSDEs. Recall that, for $u \in H^{1}\left(\mathbb{R}^{d}\right)$, the martingale part of the Fukushima decomposition (2.5) is as follows

$$
\left.M u\right|_{0} ^{t}=\int_{0}^{t} \nabla u\left(X_{r}\right) d W_{r}+\int_{0}^{t} \int_{\mathbb{R}^{d}}\left[u\left(X_{r-}+z\right)-u\left(X_{r-}\right)\right] \tilde{N}(d z, d r) .
$$

Hence, by Theorem 4.10, if $u \in \mathcal{H}_{T}$ is the solution for stochastic PIDE (1.1), we have

$$
\begin{aligned}
u_{t}\left(X_{t}\right)= & \Phi\left(X_{T}\right)-\int_{t}^{T} \nabla u_{r}\left(X_{r}\right) d W_{r}-\int_{t}^{T} \int_{\mathbb{R}^{d}} u_{r}\left(X_{r-}+z\right)-u_{r}\left(X_{r-}\right) \tilde{N}(d z, d r) \\
& +\int_{t}^{T} f_{r}\left(X_{r}, u_{r}\left(X_{r}\right), \nabla u_{r}\left(X_{r}\right)\right) d r+\int_{t}^{T} h_{r}\left(X_{r}, u_{r}\left(X_{r}\right), \nabla u_{r}\left(X_{r}\right)\right) \overleftarrow{d B}_{r} \\
& -\int_{t}^{T} g_{r}\left(X_{r}, u_{r}\left(X_{r}\right), \nabla u_{r}\left(X_{r}\right)\right) * d X, \quad \mathbb{P}^{\prime} \otimes \mathbb{P}-\text { a.s.. }
\end{aligned}
$$

Setting

$$
Y_{t}:=u_{t}\left(X_{t}\right), \quad Z_{t}:=\nabla u_{t}\left(X_{t}\right), \quad U_{t}(z):=u_{t}\left(X_{t-}+z\right)-u_{t}\left(X_{t-}\right),
$$

we find that the triplet of processes $(Y, Z, U)$ satisfies the following equation

$$
\begin{aligned}
Y_{t}=\Phi\left(X_{T}\right) & -\int_{t}^{T} Z_{r} d W_{r}-\int_{t}^{T} \int_{\mathbb{R}^{d}} U_{r}(z) \tilde{N}(d z, d r)+\int_{t}^{T} h_{r}\left(X_{r}, Y_{r}, Z_{r}\right) \overleftarrow{d B}_{r} \\
& +\int_{t}^{T} f_{r}\left(X_{r}, Y_{r}, Z_{r}\right) d r-\int_{t}^{T} g_{r}\left(X_{r}, Y_{r}, Z_{r}\right) * d X
\end{aligned}
$$

Definition 2. (1) In the case that $h=0$, if $w(t, x) \in L^{2}\left([0, T] \times \mathbb{R}^{d}\right), \phi \in L^{2}([0, T] \times$ $\left.\mathbb{R}^{d}, \mathbb{R}^{d}\right) ; \psi \in L^{2}\left([0, T] \times \mathbb{R}^{d} ; L^{2}\left(\mathbb{R}^{d}, d \nu\right)\right)$, setting $Y_{t}:=w\left(t, X_{t}\right), Z_{t}:=\phi\left(t, X_{t}\right)$ and $U_{t}(z):=$ $\psi\left(t, X_{t-}, z\right)$, we say that $(Y, Z, U)$ is a triplet of solution to the nonlinear BSDEs with terminal condition $\xi=\Phi\left(X_{T}\right)$ and coefficients $f, g$ satisfying assumptions (H) and (HD2), provided the following relation holds

$$
\begin{aligned}
Y_{t}=\xi-\int_{t}^{T} Z_{r} d W_{r}-\int_{t}^{T} \int_{\mathbb{R}^{d}} U_{r}(z) \tilde{N}(d z, d r)+\int_{t}^{T} f_{r}\left(X_{r}, Y_{r}, Z_{r}\right) d r \\
-\int_{t}^{T} g_{r}\left(X_{r}, Y_{r}, Z_{r}\right) * d X, \quad \text { for } t \in[0, T], \quad \mathbb{P}^{\prime} \otimes \mathbb{P}-\text { a.s.. }
\end{aligned}
$$

(2) In the case that $h=\left(h_{1}, \ldots, h_{d^{1}}\right): \Omega^{\prime} \times[0, T] \times \mathbb{R}^{d} \times \mathbb{R} \times \mathbb{R}^{d} \rightarrow \mathbb{R}^{d^{1}}$, if $w \in L^{2}\left(\Omega^{\prime} \times\right.$ $\left.[0, T] \times \mathbb{R}^{d}\right), \phi \in L^{2}\left(\Omega^{\prime} \times[0, T] \times \mathbb{R}^{d}, \mathbb{R}^{d}\right) ; \psi \in L^{2}\left(\Omega^{\prime} \times[0, T] \times \mathbb{R}^{d} ; L^{2}\left(\mathbb{R}^{d}, d \nu\right)\right)$, defining $Y_{t}=$ $Y_{t}\left(\omega^{\prime}, \omega\right):=w\left(\omega^{\prime}, t, X_{t}(\omega)\right), Z_{t}=Z_{t}\left(\omega^{\prime}, \omega\right):=\phi\left(\omega^{\prime}, t, X_{t}(\omega)\right)$ and $U_{t}(z)=U_{t}\left(\omega^{\prime}, \omega, z\right):=$ $\psi\left(\omega^{\prime}, t, X_{t-}(\omega), z\right)$, we say that $(Y, Z, U)$ is a solution of the nonlinear BDSDEs with 
terminal condition $\Phi\left(X_{T}\right)$ and coefficients $f, g, h$ satisfying assumptions (H) and (HD2), provided that the relation (5.2) holds.

It has been proved that the triplet of processes $(Y, Z, U)$, defined in (5.1) with $u \in \mathcal{H}_{T}$ being the solution of equation (1.1), is the solution of non-linear BDSDE (5.2). Actually, the converse statement can also be proved. We therefore build a connection between the solutions for stochastic PIDEs and BDSDEs.

Theorem 5.1. Suppose $Y_{t}=w\left(t, X_{t}\right), Z_{t}=\phi\left(t, X_{t}\right), U(t, z)=\psi\left(t, X_{t-}, z\right)$ is a solution of the non-linear BDSDE (5.2) defined as Definition 2 (2), then $w \in \mathcal{H}_{T}$ represents a weak solution of stochastic PIDE (1.1) as defined in Definition 4.1.

Proof. We only give the proof in the linear case, i.e. $f, g, h$ only depend on $(t, x)$, and the nonlinear case follows easily.

Suppose $w^{\prime} \in \mathcal{H}_{T}$ is a solution of stochastic $\operatorname{PIDE}(1.1)$, then we have $Y_{t}^{\prime}=w^{\prime}\left(t, X_{t}\right)$, $Z_{t}^{\prime}=\nabla w^{\prime}\left(t, X_{t}\right), U^{\prime}(t, z)=w^{\prime}\left(t, X_{t-}+z\right)-w^{\prime}\left(t, X_{t-}\right)$ is a solution for BDSDE (5.2). By linearity, setting $\bar{Y}=Y-Y^{\prime}, \bar{Z}=Z-Z^{\prime}$ and $\bar{U}=U-U^{\prime}$, we have $\mathbb{P}^{\prime} \otimes \mathbb{P}$-almost surely,

$$
\bar{Y}_{t}=-\int_{t}^{T} \bar{Z}_{s} d W_{s}-\int_{t}^{T} \int_{\mathbb{R}^{d}} \bar{U}_{s}(z) \tilde{N}(d z, d s) .
$$

Applying Itô's formula and taking expectation under $\mathbb{P}^{\prime} \otimes \mathbb{P}$, it follows that

$$
\left|\bar{Y}_{t}\right|^{2}+\int_{t}^{T}\left|\bar{Z}_{s}\right|^{2} d s+\int_{t}^{T} \int_{\mathbb{R}^{d}}\left|\bar{U}_{s}(z)\right|^{2} \nu(d z) d s=0, \quad \mathbb{P}^{\prime} \otimes \mathbb{P}-\text { a.s. },
$$

which implies that $Y_{t}=Y_{t}^{\prime}, \mathbb{P}^{\prime} \otimes \mathbb{P}-$ a.s., then $w(t, \cdot)=w^{\prime}(t, \cdot), d \mathbb{P}^{\prime} \otimes d x-$ a.e.; $Z_{t}=$ $Z_{t}^{\prime}, \mathbb{P}^{\prime} \otimes \mathbb{P}-$ a.s., so that $\phi(t, \cdot)=\nabla w^{\prime}(t, \cdot), d \mathbb{P}^{\prime} \otimes d x-$ a.e.. Hence $w=w^{\prime} \in \mathcal{H}_{T}$, $d \mathbb{P}^{\prime} \otimes d t \otimes d x-$ a.e..

Remark 5.2. If we let the constant $a$ approaches to 0 , the operator $\mathcal{A}$ degenerates into a diffusion operator, which is associated with a continuous Markov process. In this situation, the zero-energy function

$$
\int_{s}^{t} \nabla u * d X=\left.M u\right|_{s} ^{t}+\left.\bar{M} u\right|_{s} ^{t}+\frac{\left\langle\nabla u, \nabla p_{r}^{\mu}\right\rangle}{p_{r}^{\mu}}\left(X_{r}\right) d r
$$

coincides with the *-integral defined in [18] and the integrand can be generalized from the gradient of functions in $H^{1}\left(\mathbb{R}^{d}\right)$ to all of the functions in $L^{2}\left(\mathbb{R}^{d}\right)$. From this point of view, we actually generalized the results in [18] for non-local operators.

\section{References}

[1] Chen, Z. Q. and Zhao, Z.: Diffusion processes and second order elliptic operators with singular coefficients for lower order terms. Math. Ann., 302(2) (1995), 323-357. MR-1336339

[2] Chen, Z. Q. and Hu, E. Y.: Heat kernel estimates for $\Delta+\Delta^{\frac{\alpha}{2}}$ under gradient perturbation. Stoch. Proc. Appl., 125(7) (2015), 2603-2642. MR-3332849

[3] Chung, K. L. and Zhao, Z.: From Brownian motion to Schrödinger's equation. Springer, Berlin (1995). MR-1329992

[4] Denis, L.: Solutions of stochastic partial differential equations considered as Dirichlet Processes. Bernoulli, 10(5) (2004), 783-827. MR-2093611

[5] Denis, L. and Stoica, L.: A general analytical result for non-linear SPDE's and applications. Electron. J. Probab., 9(23) (2004), 674-709. MR-2110016

[6] Fukushima, M., Oshima, Y. and Takeda, M.: Dirichlet forms and symmetric Markov processes. De Gruyter Studies in Mathematics, 19. Walter de Gruyter \& Co., Berlin (1994). MR-1303354

[7] Kwaśnicki, M.: Ten equivalent definitions of the fractional Laplace operator. Fract. Calc. Appl. Anal., 20(1) (2017), 7-51. MR-3613319 
[8] Lyons, T. J. and Stoica, L.: The limits of stochastic integrals of differential forms. Ann. Probab., 27(1) (1999), 1-49. MR-1681146

[9] Lyons, T. J. and Zhang, T. S.: Decomposition of Dirichlet processes and its application. Ann. Probab., 22(1) (1994), 494-524. MR-1258888

[10] Lunt, J., Lyons, T. J. and Zhang, T. S.: Integrability of functionals of Dirichlet processes, probabilistic representations of semigroups, and estimates of heat kernels. J. Funct. Anal., 153(2) (1998), 320-342. MR-1614598

[11] Ma, Z. M. and Röckner, M.: Introduction to the theory of (nonsymmetric) Dirichlet forms. Springer-Verlag, Berlin, 1992. MR-1214375

[12] Oshima, Y.: Semi-Dirichlet forms and Markov processes. De Gruyter Studies in Mathematics, 48. Walter de Gruyter \& Co., Berlin, 2013. MR-3060116

[13] Pardoux, É. and Peng, S.: Adapted solution of a backward stochastic differential equation. Systems Control Lett., 14(1) (1990), 55-61. MR-1037747

[14] Pardoux, É. and Peng, S.: Backward stochastic differential equations and quasilinear parabolic partial differential equations. Stochastic partial differential equations and their applications (Charlotte, NC, 1991), 200-217, Lect. Notes Control Inf. Sci., 176, Springer, Berlin, 1992. MR-1176785

[15] Pardoux, É. and Peng, S.: Backward doubly stochastic differential equations and systems of quasilinear SPDEs. Probab. Theory Related Fields, 98(2) (1994), 209-227. MR-1258986

[16] Pardoux, É.: Backward stochastic differential equations and viscosity solutions of systems of semilinear parabolic and elliptic PDEs of second order. Stochastic analysis and related topics, VI (Geilo, 1996) 79-127, Progr. Probab., 42, Birkhäuser Boston, Boston, MA, 1998. MR-1652339

[17] Protter, P.: Volterra equations driven by semimartingales. Ann. Probab., 13(2) (1985), 519530. MR-0781420

[18] Stoica, I. L.: A probabilistic interpretation of the divergence and BSDE's. Stoch. Proc. Appl., 103(1) (2003), 31-55. MR-1947959

[19] Sun, W.: The complement value problem for non-local operators. arXiv:1804.00212.

[20] Tartar, L.: An Introduction to Sobolev Spaces and Interpolation Spaces. Lecture Notes of the Unione Matematica Italiana, 3. Springer, Berlin; UMI, Bologna, 2007. MR-2328004

Acknowledgments. The authors would like to thank the anonymous referees for the careful reading and very useful comments. 